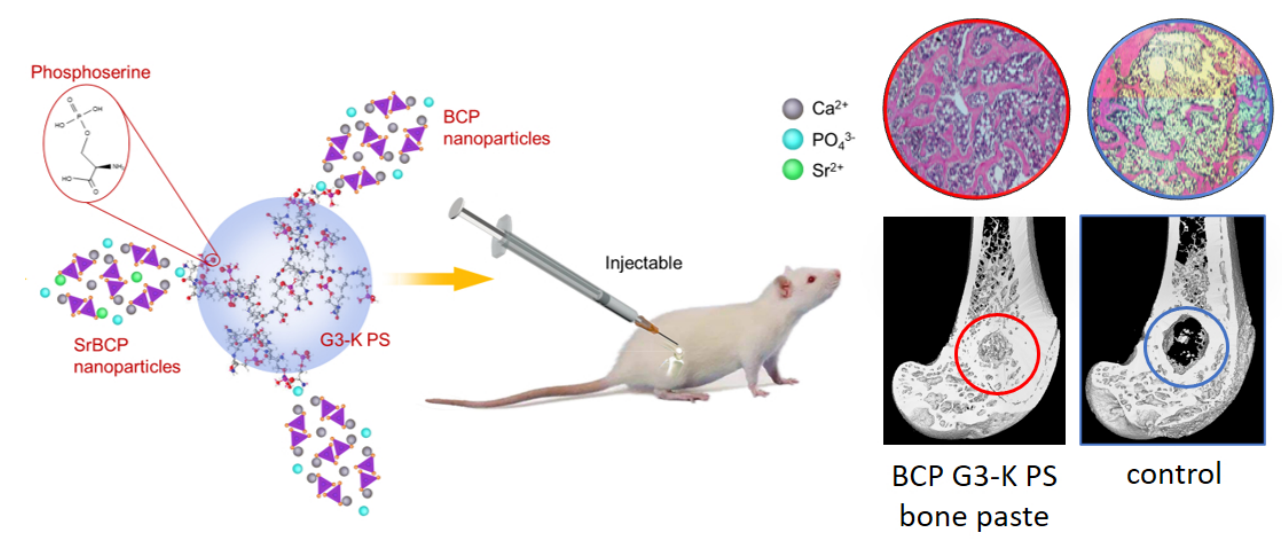

Schematic illustration of Injectable biphasic calcium phosphates with osteoinductive properties $48 \times 19 \mathrm{~mm}(600 \times 600 \mathrm{DPI})$ 


\title{
Complexation of Injectable Biphasic Calcium Phosphate with Phosphoserine- Presenting Dendrons with Enhanced Osteoregenerative Properties
}

\author{
Long Yang ${ }^{1}$, Siyu Chen ${ }^{1}$, Tieliang Shang ${ }^{1}$, Rui Zhao ${ }^{1}$, Bo Yuan ${ }^{1}$, Xiangdong Zhu ${ }^{1}$, \\ Maria Grazia Raucci ${ }^{2, *}$, Xiao Yang ${ }^{1, *}$, Xingdong Zhang ${ }^{1}$, Matteo Santin ${ }^{3}$, Luigi \\ Ambrosio $^{2}$
}

${ }^{1}$ National Engineering Research Center for Biomaterials, Sichuan University, Chengdu 610064, China

${ }^{2}$ Institute of Polymers, Composites and Biomaterials (IPCB) - National Research Council of Italy (CNR), 80125 Naples, Italy

${ }^{3}$ School of Pharmacy \& Biomolecular Sciences, University of Brighton, Huxley Building Lewes Road Brighton BN2 4GJ UK

*Corresponding authors:

Dr. Maria Grazia Raucci

Email: mariagrazia.raucci@cnr.it

\section{Dr. Xiao Yang}

Email: xiaoyang114@scu.edu.cn 


\begin{abstract}
Injectable biphasic calcium phosphates have been proposed as a solution in the treatment of a range of clinical applications including as fillers in the augmentation of osteoporotic bone. Until now, various biodegradable natural or synthetic organics have been used as polymer component of bone materials to increase their cohesiveness. Herein, a novel bone material was developed combining osteoconductive biphasic calcium phosphate (BCP) nanoparticles with phosphoserine-tethered generation 3 poly(epsilon-lysine) dendron (G3-K PS), a class of hyperbranched peptides previously shown to induce biomineralization and stem cell osteogenic differentiation. Strontium was also incorporated into the $\mathrm{BCP}$ nanocrystals $(\mathrm{SrBCP})$ to prevent bone resorption. Within 24 hours, an anti-washout behavior was observed in G3-K PS integrated pure BCP group (BCPG3). Moreover, both in vitro tests by relevant cell phenotypes and an in vivo tissue regeneration study by an osteoporotic animal bone implantation showed that the integration of G3-K PS would downregulate $\mathrm{Cxcl} 9$ gene and protein expressions thus enhancing bone regeneration measured as bone mineral density, new bone volume ratio and trabecular microarchitectural parameters. However, no synergistic effect was found when $\mathrm{Sr}$ was incorporated in to the BCPG3 bone pastes. Noticeably, the results indicated a concomitant reduction of bone regeneration potential assessed as reduced Runx 2 and PINP expression when bone resorptive RANKL and CTX-I levels were reduced by Sr supplementation. Altogether, the results suggest the potential of injectable BCPG3 bone materials in the treatment of osteoporotic bone defects.
\end{abstract}


KEYWORDS: injectability, strontium, dendrimer, osteoporosis, biphasic calcium phosphate

\section{INTRODUCTION}

Clinical application of injectable bone pastes has been increasing rapidly in recent decades because of their advantages including ease to adaptation into irregularstructured defects (e.g. absence of gaps between the host bone and the implanted filler) and injectability suitable to minimally invasive surgery. These advantages are widely advocated to reduce patient complications and health care costs. ${ }^{1,2}$ Several widely used injectable bone materials are summarized in Table 1. Moreover, biodegradable injectable bone pastes have shown significant bone regeneration potential in many clinical conditions, such as the treatment of maxillofacial deformities and defects, certain indications of spinal fusion and augmentation of osteoporotic fractures. ${ }^{3-6}$ Various biodegradable natural or synthetic hydrogels have been used as excipients or carriers components in injectable bone pastes. Poly(L-lactic acid), polycaprolactone (PCL), poly(lactide-co-glycolide), polyethylene glycol (PEG) and their copolymers are examples of synthetic carriers whereas collagen, hyaluronic acid, sodium alginate, chitosan and gelatin are the most prevalent natural carries. ${ }^{7-9}$ These hydrogels are able to improve pastes flexibility and cohesiveness and also offer a physical support, a biodegradable hydrated extracellular matrix which affects the cell-scaffold interactions, biologically and physically modulating cell invasion and proliferation in the construct. ${ }^{10}$ Although these hydrogels may alone be employed as injectable 
biomaterials, lacks of osteogenic ability limits their use of this form. Therefore, different osteoconductive inorganic granules such as hydroxyapatite (HA), betatricalcium phosphate $(\beta-\mathrm{TCP})$, biphasic calcium phosphate $(\mathrm{BCP}$, a combination of HA and $\beta$-TCP) and bioactive glass have been used to combine with these hydrogels and improve in situ bone regeneration. ${ }^{11-14}$

Significant efforts have been expended towards exploring the optimal formulation of the bone pastes in recent years. Salehi et al. demonstrated the possibility to develop different injectable materials from gelatin, carboxymethyl cellulose and alginate as carrier phase and BCP as osteoinductive phase. ${ }^{15}$ Stability, injectability, washout and the rheology performances of materials were evaluated. The results proved that the polymers in higher amount may modulate the degradation rate of materials and improve the injectability at lower load. Liu et al. prepared injectable bone paste at different compositions based on different sizes of HA particles and sodium hyaluronate solution. ${ }^{15}$ All the materials were injected by applying a load in the range of $10-20 \mathrm{~N}$, while higher load was requested in the presence of smaller HA particles size. Wei et al. fabricated injectable HA/collagen pastes loaded with or without bone morphogenetic proteins-2 (BMP-2). ${ }^{16} \mathrm{~A}$ rat femur defect model was used to investigate bone healing capacity of the bone pastes. Eight weeks' post-surgery, micro-CT scanning demonstrated that bone union was achieved in all bone paste implanted groups, not in the non-treated control group. The mechanical test and histological evaluation further indicated that HA/collagen paste with or without BMP-2 augmented bone union. 
Dendrimers are highly ordered hyperbranched, three-dimensional polymers forming nanostructures, which are synthesized by coupling different monomers able to grow into branching polymers. Indeed, dendrimers may be developed from synthetic molecules (e.g. polyamido amine) as well as from amino acids (e.g. poly-L-lysine) and carbohydrates. ${ }^{17-20}$ Poly(epsilon-lysine) dendrimers are well-known cell-adhesion enhancer and their presence is expected to promote cellular invasion and further bioactivity to the implant. ${ }^{21-23}$ In regard to bone regeneration, a phosphoserine(PS)tethered generation 3 poly(epsilon-lysine) dendron (G3-K PS) has been developed. The last generation ( $3^{\text {rd }}$ generation) of this dendron is bioactivated by phosphoserine which catalyzes the biomineralization process in living tissues. When incorporated in the structure of proteins and cell membrane, this phosphorylated amino acid is able to catalyze the formation of apatite crystals. ${ }^{24,25}$ Previously, G3-K PS films were generated and deposited on implantable titanium or titanium oxide surfaces. ${ }^{17,26}$ The G3-K PS dendron films showed the capability to stimulate an uniform deposition of calcium phosphate phase in a simulated body fluid. The results of cell culture studies using osteoblast and bone marrow stromal cells (MSCs) demonstrate a good viability of the cells on the G3-K PS-modified materials. Furthermore, the cell proliferation and differentiation through osteoblastic phenotype were found to be enhanced.

Here, sol-gel method was used to develop an injectable bone paste integrating BCP nanoparticles (HA: $\beta-\mathrm{TCP}=2: 8)$ with $\mathrm{G} 3-\mathrm{K}$ PS. In our previous study, BCP nanoparticles were shown to enhance osteointegration of the bioceramic implant in segmental defect of beagle dogs and rats. ${ }^{27,28}$ In this work, the incorporation of 
strontium ( $\mathrm{Sr}$ ) element into the BCP nanocrystals was also considered to minimize bone resorption. It was widely observed that $\mathrm{Sr}$ element have an inhibitory effect on osteoblast resorbing activity. ${ }^{29,30}$ Number and size of the resorption pits would be decreased when Sr was incorporated. ${ }^{31}$ At molecular level, osteoclastogenesis could be retarded by Sr through downregulating nuclear factor- $\kappa \mathrm{B}(\mathrm{NF}-\kappa \mathrm{B})$ signal pathway. ${ }^{32,33}$ The physio-chemical properties and injectable capability of the fabricated material were first characterized. Then, in vitro cell proliferation and differentiation of the MSCs isolated from osteoporotic bone cocultured with different bone pastes were analyzed. Additionally, the in vivo performance of materials was investigated by using an ovariectomized rat critical-sized femoral bone defect model. The osteogenic property of bone pastes was evaluated with micro-CT and histological analysis.

\section{EXPERIMENTAL SECTION}

\subsection{Fabrication of the bone pastes}

The schematic illustration of the fabrication progress for bone pastes was shown Fig. 1A. The preparation of materials including BCP, SrBCP (with $15 \mathrm{~mol} \% \mathrm{Ca}^{2+}$ replaced by $\mathrm{Sr}^{2+}$ ), BCPG3 (BCP in G3-K PS carrier) and SrBCPG3 (SrBCP in G3-K PS carrier) was adapted from previously optimized protocols on SrHA and HA/G3-K PS preparation. ${ }^{21,34} \mathrm{BCP}$ was synthesized at room temperature using calcium nitrate tetrahydrated $\mathrm{Ca}\left(\mathrm{NO}_{3}\right)_{2} \cdot 4 \mathrm{H}_{2} \mathrm{O}$ (Sigma-Aldrich, China) and di-ammonium hydrogen phosphate $\left(\mathrm{NH}_{4}\right)_{2} \mathrm{HPO}_{4}$ (Sigma-Aldrich, China) as precursors and distilled water as solvent. For the preparation of $\mathrm{SrBCP}$, strontium nitrate $\mathrm{Sr}\left(\mathrm{NO}_{3}\right)_{2}$ (Sigma-Aldrich, 
China) was added in distilled water to replace $15 \mathrm{~mol}^{\circ} \mathrm{Ca}^{2+}$. Particularly, the process consists of mixing of a $3 \mathrm{M}\left(\mathrm{NH}_{4}\right)_{2} \mathrm{HPO}_{4}$ solution to a $4.6 \mathrm{M} \mathrm{Ca}^{2+}$ or $\mathrm{Ca}^{2+}-\mathrm{Sr}^{2+}$ solution under stirring at $200 \mathrm{rpm}$ and $40{ }^{\circ} \mathrm{C}$. The $\mathrm{pH}$ of the mixed solution was continuously monitored by a $\mathrm{pH}$ meter and adjusted to 9 by adding $1.5 \mathrm{~mL}$ ammonium hydroxide. G3-K PS semi-dendrimers were synthesized by a solid-phase peptide synthesis with a microwave synthesizer (Biotage Initiator) as previously reported. ${ }^{21}$ Freeze-dried G3-K PS semi-dendrimers in ethanol solution $\left(5 \mathrm{mg} \mathrm{mL}^{-1}\right)$ was added at $1 \%(\mathrm{w} / \mathrm{w})$ to the above $\mathrm{BCP}$ or SrBCP slurry $(\mathrm{Ca}+\mathrm{Sr} / \mathrm{P}$ in the range of 1.53$)$ under $200 \mathrm{rpm}$ and $37^{\circ} \mathrm{C}$ stirring until gelation occurred. The gels were then dialyzed in $0.01 \mathrm{M}$ phosphate buffered saline (PBS) at $\mathrm{pH} 7.4$ until equilibrated to the buffer $\mathrm{pH}$.

\subsection{Material characterization of the injectable bone pastes}

The morphology and crystal size of the nanoparticles in the material paste were evaluated via transmission electron microscopy (TEM, ZEISS Libra 200 FE, Germany) using an accelerating voltage at $200 \mathrm{kV}$. All the samples were diluted in deionized water and mixed by slightly shaking, and then dispersed by ultrasonic waves. For TEM images, a droplet of diluted paste suspensions was placed onto carbon coated copper grids and dried in air at room temperature.

The phase composition of the materials was analyzed using X-ray diffraction (XRD, Empyrean PANalytical, Netherlands) with $\mathrm{Cu} \mathrm{K \alpha}$ radiation. The pastes were freezedried and milled, and then passed through a 200-mesh stainless steel screen to obtain the fine powder. Then, the powders were located in a constant temperature oven 
$\left(\mathrm{T}=37^{\circ} \mathrm{C}\right)$ until a constant weight was obtained. Samples were scanned at accelerating voltage of $40 \mathrm{kV}$ and current of $35 \mathrm{~mA}$. Diffraction patterns were collected from 20 to $60^{\circ}(2 \theta)$. The step size was $0.026^{\circ}(2 \theta)$ with a scan rate of $14^{\circ}(2 \theta)$ per minute. The XRD results were matched with a standard card for HA (ICDD PDF card \#9-432) and $\beta$-TCP (ICDD PDF card \#9-169) to discern the difference in phase composition. Fourier transform infrared spectroscopy (FTIR, Nicolet 6700, Thermo, USA) was also used to determine chemical composition of the pastes same as those examined by XRD as mentioned above.

\subsection{Injectability and washout test of the bone pastes}

The handling properties of the pastes were analyzed on the basis of their injectability and moulding. In this way, the materials were moved into a syringe (model and needle size) and manually injected into deionized polystyrene sponge at $37^{\circ} \mathrm{C}$. Visual check of the shape change of the materials was then performed. Moreover, the washout resistance of the pastes was evaluated by a protocol developed in previous research. ${ }^{30,35}$ In brief, freshly made bone pastes were loaded into a syringe and then injected into a PBS solution or cell culture medium at $37{ }^{\circ} \mathrm{C}$. After 1 hour, 24 hours, and 48 hours of immersion, the percentage amount of materials being washed out was quantitatively measured. Calcium and strontium ion release profiles in PBS and culture medium during 7 days of material degradation were also examined by an inductively 
coupled plasma optical emission spectrometer (ICP-OES, SPECTRO ARCOS, Germany). The tests were executed in triplicate unless differently reported.

\subsection{Isolation and MSCs from osteoporotic rats}

In vitro cell tests to assess the osteogenic potential of the synthesized biomaterials were performed using bone marrow stromal cells MSCs isolated from ovariectomized rats. The isolation and culture protocols were adapted from a previous study. ${ }^{36}$ The cells were collected from the femurs of three randomly selected rats 2 months after ovariectomy. Under standard sterile conditions, the femur bones were washed with $1 \%$ (w/v) PBS three times and dissected to expose the medullary canal. The bone marrow was flushed out of the cavity using Dulbecco's modified Eagle's medium (Gibco, USA) supplemented with $10 \%(\mathrm{v} / \mathrm{v})$ standard fetal bovine serum (FBS, Gibco, USA) and 1\% (w/v) penicillin/streptomycin (Gibco, USA). Then, cell suspension was filtered to eliminate any cell clusters and tissue debris. After 48 hours of incubation under standard cell culture condition at $37^{\circ} \mathrm{C}$ in a humidified atmosphere of $95 \%$ air and $5 \% \mathrm{CO}_{2}$, the non-adherent hemocytes were washed away leaving only the adherent cells to proliferate. When $90 \%$ confluent was reached, the MSCs were further passaged. The medium was replaced every 3 days. Passage 3 were used in the whole study.

Cell suspension was seeded into $24-w e l l$ plates at a density of $10^{4}$ cells per well. After cell-substrate attachment, the bone pastes in culture medium at a concentration of $100 \mathrm{mg} \mathrm{mL}^{-1}$ were kept in contact with cells for 1, 3 and 7 days. According to our 
previous protocol, ${ }^{37}$ the phenotype of isolated cells was characterized by analyzing the expression profile of lineage-specific markers via flow cytometry, demonstrating that they expressed high levels ( $>90 \%)$ of CD29 and CD90, but low levels $(<5 \%)$ of CD34 and CD45. It was confirmed that these isolated cells derived from rat bone marrow were mesenchymal types. The medium was replaced at days 3 and 7 with fresh medium containing bone pastes. The viability of MSCs grown on different bone pastes was evaluated using the cell counting kit-8 (Dojindo, Kamimashiki-gun, Kumamoto, Japan) at day 1, 3, and 7 by microplate reader (Varioskan Flash, Thermo Scientific, USA) as reported in a previous study. ${ }^{37}$ The cell-materials interaction was observed by confocal laser scanning microscopy (CLSM, LeicaTCS-SP5, Germany) at day 3 by using FDA/PI staining.

\subsection{Osteoblastic gene expression and Western blotting analyses}

After 3 and 5 days of coculture, the cells were detached and centrifuged at 10000 rpm for 5 min for RNA isolation and quantification. The samples were gently washed three times with PBS, and total RNA was extracted using the RNeasy Mini Kit (Qiagen, Germany). Then, concentration and purity of the total RNA were measured by using a Nanodrop 2000 UV spectrophotometer (Thermo Scientific, USA). An iScript ${ }^{\mathrm{TM}}$ cDNA Synthesis Kit (Bio-Rad, CA, USA) was used to reverse-transcribe the total RNA into complementary DNA (cDNA) in a $20-\mu \mathrm{L}$ reaction system. A CFX-96t real-time PCR detection system (CFX960, Bio-Rad, USA) with SsoFast EvaGreens Supermix (BioRad, USA) was employed to perform real-time quantitative PCR reaction. In particular, the amplification procedure was an incubation at $95^{\circ} \mathrm{C}$ for $2 \mathrm{~min}$, followed by 40 cycles 
of $95^{\circ} \mathrm{C}$ for $2 \mathrm{~s}$ and $60^{\circ} \mathrm{C}$ for $5 \mathrm{~s}$. The relative mRNA expression level of each gene was expressed as threshold cycle values and GAPDH was selected as the housekeeping gene to normalize the results. The gene expression levels of runt-related transcription factor 2 (Runx2), alkaline phosphatase (ALP), C-X-C motif chemokine ligand 9 (Cxc19), receptor activator of NF- $\mathrm{B}$ ligand (RANKL) were measured.

Western blotting investigation of the proteins with regard to differentially expressed genes was conducted according to our previous method. ${ }^{38}$ Furthermore, to confirm the role of $\mathrm{Cxc19}$ and $\mathrm{Sr}$ ions in material induced osteogenesis, additional $\mathrm{Cxc19}$ protein (PrimeGene Bio-Tech, \#221-09, $250 \mathrm{ng} \mathrm{mL}^{-1}$ ) was introduced into the BCPG3 and $\mathrm{SrBCP}$ groups in culture medium, and $\mathrm{Sr}$ ions $(3 \mathrm{mM})$ were introduced into the BCP and BCPG3 groups in cell culture medium, in the samples prepared for Western blotting. The primary antibodies to Runx2, RANKL, GAPDH were acquired from Cell Signalling, MA, USA. The protein levels were analyzed by using the BCA protein assay kit (Pierce of Thermo Fisher Scientific Inc., Rock- ford, IL). Equal amounts of protein were separated by sodium dodecyl sulfate-polyacrylamide gel electrophoresis and transferred to membranes. Protein bands were envisioned using a ChemiDocTM XRS+ image system with image $\mathrm{Lab}^{\mathrm{TM}}$ software (Bio-Rad, USA).

\subsection{In vivo implantation}

All animal procedures in the experiment were executed in according with the Guidelines for Care and Use of Laboratory Animals of Sichuan University and tests were accepted by the Animal Care and Use Committee of Sichuan University. Twenty- 
four female Sprague Dawley rats $(235 \pm 8$ g, 12 weeks old, Chengdu Dashuo Experimental Animal Co. Ltd, China) were subjected to ovariectomy surgery and then housed for 2 months to induce osteoporotic conditions. ${ }^{29,39}$ After 8 weeks, the rats were randomly divided into the four bone paste groups including $\mathrm{BCP}$ (BCP in $\mathrm{PBS}$ carrier), SrBCP (SrBCP in PBS carrier), BCPG3 (BCP in G3-K PS carrier) and SrBCPG3 (SrBCP in G3-K PS carrier). A defect of $3.0 \mathrm{~mm}$ in diameter and $4.0 \mathrm{~mm}$ in depth centered on the distal femur axis of the rats was created using a dental drill as described in our previous work. ${ }^{27}$ Different bone pastes were gently injected into the drilled holes by syringe. The rats were euthanized at week 12 post implantation by an intraperitoneal overdose injection of pentobarbital. Blood and femur samples were harvested. Prior to the further analysis, the harvested femur specimens were immediately fixed in $4 \%$ paraformaldehyde for 1 week and the blood was centrifuged to obtain the upper serum for 15 minutes at $1000 \times \mathrm{g}$ at $4{ }^{\circ} \mathrm{C}$. Several bone metabolic markers were evaluated using commercial enzyme-linked immunosorbent assay (ELISA) kit, including procollagen I N-terminal peptide (P1NP, E90957, Uscnlife, China), cross-linked Ctelopeptide of type I collagen (CTX-I, cat. E0665m, Uscnlife, China) and Cxc19 (Cxc19, E1928Ra, Uscnlife, China). The measurement procedures were performed in accordance with manufacturer's instructions. The concentrations of the markers were calculated according to the standard curve. Furthermore, the hematological parameters, including white blood cell count (WBC), lymphocytes (LYM), monocytes (MONO), neutrophils (NEUT), erythrocytes (RBC), haemoglobin (HGB) and platelet count (PLT) were measured by a hematological autoanalyzer (ADVIA 2120i, Siemens, Germany). 


\subsection{Micro-computed tomography (micro-CT) analysis}

micro-CT system ( $\mu$ CT80, Scanco Medical, Basersdorf, Switzerland) was used to evaluate new bone formation within the implants. The X-ray tube was set at $70 \mathrm{kVp}$ and $114 \mathrm{~mA}$. A resolution of $20 \mu \mathrm{m}$ per pixel was set. The acquired greyscale images were reconstructed and analyzed using Mimics Research 17.0 (Materialise Co., Belgium). A threshold was applied to differentiate non-degraded bone paste material from the newly formed bone tissue according to our previously established method. ${ }^{27,38}$ After thresholding, the newly formed bone within the defected region was quantitatively determined by normalizing the calculated bone volume to the total volume of the defect area (BV/TV). Moreover, direct measurements with Scanco analytical software (SCANCO VivaCT80, Switzerland) were used to evaluate trabecular density and architectural parameters within the defect, including bone mineral density (BMD), structural model index (SMI), trabecular number (Tb.N), trabecular thickness (Tb.Th) and trabecular separation (Tb.Sp).

\subsection{Histological staining}

After micro-CT analysis, the implanted samples derived from different groups were decalcified in $10 \%$ ethylenediaminetetraacetic acid at room temperature for 6 weeks. The solution was changed 3 times a week. Then, the tissues were washed and dehydrated with ethanol solutions at different concentrations (from 50 to $100 \mathrm{v} / \mathrm{v} \%$ ), cleared in xylene and embedded in paraffin. Sections of $5 \mu \mathrm{m}$ thickness were obtained 
by a microtome and mounted on glass slides. Next, the transverse sections were stained with hematoxylin and eosin (H\&E) as a conventional histologic method to observe new bone formation. For immunohistochemistry, sections were dewaxed and gradient hydrated. After blocking with 5\% hydrogen peroxidase for $5 \mathrm{~min}$, the sections were incubated with primary antibodies against Cxc19 (1:400), Runx2 (1:400) and RANKL (1:300) for $12 \mathrm{~h}$ (Cell Signaling, MA, USA). After washing, the sections were further incubated with secondary antibodies and then avidin-biotin enzyme reagents for $30 \mathrm{~min}$, followed by counterstaining with hematoxylin. Each slide was observed under light microscope (Bx60, Olympus, Japan) equipped with a digital CCD camera. Image Pro Plus 6.0 analysis software was used to quantitatively calculate the mean optical density (MOD) of Cxc19, Runx2 and RANKL positive staining area and lacuna number. In addition, part of the transverse section specimens without staining were mounted on aluminum stubs and sputter coated with a gold layer for scanning electron microscopy observation (SEM, S4800, Hitachi, Japan).

\subsection{Statistical analysis}

All experiments were executed in triplicates unless differently reported. All data are presented as the mean \pm standard deviation (mean $\pm \mathrm{SD}$ ). Statistical analysis of the data was performed using SPSS11.0 software. The observed differences between the groups were determined using one-way ANOVA followed by Tukey's multiple comparison post hoc test with a level of significance of $\mathrm{p}<0.05$. 


\section{RESULTS}

\subsection{Material characterization}

As mentioned earlier, Fig. 1A illustrated the fabrication process of different bone pastes by sol-gel method at low temperature. Fig. 1B TEM images indicated that the nanoparticles in the bone pastes were approximately $15 \mathrm{~nm}$ in diameter and $80 \mathrm{~nm}$ in length. There was no observable difference between groups. The results showed that both incorporation of Sr and G3-K PS carrier exerted little influence on the morphology or particle size of BCP nanoparticles. Fig. 1C shows the XRD patterns of the four bone paste materials. The presence of both HA and $\beta$-TCP peaks were detected in the spectra of all materials. The ratio of HA to $\beta$-TCP was analyzed by the integrated intensity of the chosen specific peaks of HA (detected at $2 \theta=31.8^{\circ}$ ) and $\beta$-TCP (detected at $2 \theta$ $=31.07^{\circ}$ ). The ratio was approximately $20 / 80$ by weight for all bone paste groups. In accordance with our previous study, the FTIR results (Fig. 1D) revealed successful integration of G3-K PS. ${ }^{34}$ Strong amide I, II and III bands in the range of 800 to 1600 $\mathrm{cm}^{-1}$ appeared in G3-K PS integrated groups. The spectra of BCP and SrBCP presented similar characteristic bands. Bands at 962 and $1040 \mathrm{~cm}^{-1}$ correspond to P-O stretching. ${ }^{40}$

Injectability and formability of the bone paste are important operation parameters when performed by an orthopaedic surgeon. During the application process, the handson experience to inject BCP or SrBCP nanoparticles in G3-K PS carrier is like to that in the PBS carrier in a blind test. The injected pastes, whether in G3-K PS carrier or PBS carrier, could be well retained in either polystyrene sponge or PBS at $37^{\circ} \mathrm{C}$ (Fig. 2A). After 1 hour, 24 hours, and 48 hours of immersion in PBS, the percentage amount 
of materials being washed out was recorded (Fig. 2B). The incorporation of Sr would significantly increase the amount of bone pastes being degraded in the PBS solution and cell culture medium. This result was similar to our previous study which suggested that the degradation rate of the Sr-doped HA bioceramics was much faster than pure HA bioceramics. ${ }^{29}$ The Sr dopant in the crystal lattice destabilizes stability of the calcium phosphate-based material. It was also detected a reduction in degradation rate with G3-K PS carrier especially for the BCP nanoparticles. In PBS solution, at $1 \mathrm{~h}$ and $24 \mathrm{~h}$, the washout percentage of BCPG3 group was significantly lower than that of the BCP group. Similar result was observed in cell culture medium at $24 \mathrm{~h}$. As shown in Fig. 2C, with a delayed degradation rate, the amount of calcium and strontium ion released in the cell culture medium was also slower than that in the PBS solution. Calcium ionic concentration was much higher in SrBCP and SrBCPG3 groups in PBS solution. Concentration of strontium ions that the cells were exposed to in $\mathrm{SrBCP}$ and SrBCPG3 groups was in a range of 10-60 ppm.

\subsection{Cell viability and differentiation on bone pastes}

Cell viability of the MSCs grown on different bone pastes was quantified in Fig. 3A. At day 3, as compared with other groups, BCP group alone had the lowest cell viability. MSCs in all groups presented polygonal morphology and spindle-like shape on material surface, revealing that the bone pastes were non-toxic to the cells. There was no morphological difference observed in cell spreading among different groups. At day 7, MSCs grown on BCPG3 and SrBCPG3 groups had significantly higher viability 
than BCP and SrBCP group. The integration of G3-K PS yielded a positive effect. It was further observed by CLSM images that the BCPG3 and SrBCPG3 groups had more alive cells than the other two groups (Fig. 3B). Furthermore, we investigated the influence of different bone pastes on major osteogenic genes of the MSCs derived from osteoporotic bone (Fig. 3C). It is well known that Runx2 is an important transcription factor which activates the expression of the main bone matrix genes in the early stages of osteoblastic differentiation. We observed an elevated Runx2 gene expression in SrBCP, BCPG3 and SrBCPG3 groups as compared to BCP group throughout the study period. Moreover, at day 7, BCPG3 and SrBCPG3 groups had higher Runx2 gene expressions than SrBCP group. Different trend was observed in ALP gene expression. ALP gene is highly upregulated when cells differentiate in mature osteoblast. At days 3 and 7, it was shown that G3-K PS integrated two groups had higher ALP gene expressions. However, the maximum differences in ALP expression between groups were not higher than 2-fold, revealing a relatively weak effect of different bone pastes. Moreover, we have determined gene expressions of two angiogenesis and osteoclastogenesis markers, $\mathrm{Cxc19}$ and RANKL. Cxc19 is expressed by osteoblastic cells to inhibit unnecessary angiogenesis and eventually the osteogenesis, in vitro and in vivo. ${ }^{41}$ In this current study, for the first time, it was revealed that the presence of G3-K PS dendrons would decrease Cxc19 gene expression which potentially abolish the negative feedback to angiogenesis. RANKL signaling is important for terminal differentiation of monocytes/macrophages into osteoclasts to initiate bone resorption. Here, it was observed that the incorporation of $\mathrm{Sr}$ element in bone pastes would 
decrease RANKL expression of the cocultured cells. Although differences were smaller than expected, SrBCP and SrBCPG3 groups present significantly lower RANKL gene expression than $\mathrm{BCP}$ and $\mathrm{BCPG} 3$ groups.

\subsection{The role of $\mathrm{Cxcl}$ and $\mathrm{Sr}$ ion in osteogenesis induced by different bone pastes}

According to the gene expression results, we hypothesized that the downregulation of Cxc19 in BCPG3 and SrBCPG3 groups would be a possible reason for promoted osteogenesis in these two groups. Firstly, western blotting of Runx2 protein secretion from MSCs on different bone pastes was conducted to verify the material-induced osteogenesis (Fig. 4). Like to the gene expression result, BCPG3 group had the highest Runx2 protein expression followed by the SrBCP and SrBCPG3 groups. The supplementary of Cxc19 protein in cell culture medium would abolish the effect of BCPG3 and SrBCPG3 on Runx2 expression. The addition of Cxc19 would also decrease RANKL protein expression of these two groups, indicating negative role of $\mathrm{Cxcl} 9$ in both osteogenesis and osteoclastogenesis. We also hypothesized from gene expression result that the introduction of Sr would suppress osteoclastogenesis. In agreement with gene expression result, we found a decreased RANKL protein secretion of the MSCs cocultured with SrBCP and SrBCPG3. Furthermore, the supplement of Sr ions in cell culture medium of $\mathrm{BCP}$ and $\mathrm{BCPG} 3$ groups would decrease their RANKL protein expression down to the level of SrBCP and SrBCPG3 groups. 


\subsection{Serum analysis and visualization of osteoporotic bone repair by different bone pastes}

Fig. 5A showed the timeline and organization of the in vivo experiments. There was a general rising trend of in the body weight for all the groups. Biomarker analysis indicated lower serum P1NP and CTX-I levels in all biomaterials groups (Fig. 5B). However, the levels of these two serum proteins was particularly reduced in animal groups treated with $\mathrm{SrBCP}$ and SrBCPG3. High levels of P1NP in serum reflects a sign of faster bone formation, while the high content of serum CTX-I was an indicator of faster bone resorption. The result of serum P1NP and CTX-I levels seem to indicate that the presence of strontium can reduce bone turnover. In terms of serum levels of Cxc19, significant lower values were measured in the BCPG3 and SrBCPG3 groups, in line with gene expression results. The hematological data of the animals from different groups were given in Table S1 in the supplementary information. All the measured parameters including WBC, LYM, MONO, NEUT, RBC, HGB and PLT of the implanted groups showed no significant difference, compared to the control.

Cross-sectional and micro-CT reconstructed view of 12-week implanted bone samples with different pastes were shown in Fig. 5C-F. Red arrowheads indicate nonresorbed bone paste materials highlighted by the higher X-ray intensity. At the end of week 12, BCP group showed unhealed defect region with a thick pseudo-cortical bone formation around it. It is a typical foreign body reaction replacing the desired trabecular bone. While discontinuous new trabeculae were observed inside the defect of SrBCP group, a dense trabecular mesh structure was formed in the BCPG3 group. The bone 
defect region in SrBCPG3 group was filled with sparsely distributed new bone and large amount of non-resorbed material. Typical coronal sections of the femurs from different groups were reconstructed (Fig. 5D). After 12-week implantation, drilled hole was still observable in $\mathrm{BCP}$ and $\mathrm{SrBCP}$ groups, while satisfactory bone regeneration was achieved in BCPG3 and SrBCPG3 groups. The new bone ingrowths into the bone defect were separately reconstructed and are showed in Fig. 5E. As expected, more new bone tissue development with an abundant trabeculae structure within the gap was observed in both BCPG3 and SrBCPG3 groups. The corresponding H\&E sections of different groups at the same location shown in Fig. 5F further confirmed the images generated from micro-CT. Large amount of non-resorbed material (white) was embedded in the newly formed trabeculae in the SrBCPG3 group. Moreover, more newly formed blood vessels could be found in bone matrix of BCPG3 and SrBCPG3 groups (yellow arrow).

\subsection{Microarchitecture of newly formed trabecular bone within the defect}

The quantity and quality of new bone tissue inside the defect zone was further quantified by micro-CT (Fig. 6). The transverse sections of 3D constructed micro-CT scanning of metaphyseal femur with defect region at week 8 and 12 were shown in Fig. 6A. The threshold used in the experiment excluded the areas occupied by non-resorbed materials thus measuring only the bony tissue. As shown in Fig. 6B, BMD of the BCPG3 group was significantly higher than other groups throughout the study. At week 8, BV/TV of the Sr doped or G3-K PS integrated groups was all significantly higher 
than BCP group. At week 12, BCPG3 group presented the highest BV/TV among all. New bone volume fraction (nBV/TV) within the defected area was also quantified (Fig. S1). nBV/TV of BCPG3 and SrBCPG3 groups were all higher than BCP and SrBCP groups at week 8 and week 12. BCPG3 group presented the highest nBV/TV value at week 12. A lower SMI value indicates healthier plate-like trabeculae structure. BCPG3 and SrBCPG3 groups demonstrated a significantly lower SMI than BCP group at week 12. Tb.N of the BCP and BCPG3 groups was observed to be higher than other two groups. No significant differences in Tb.Th were observed among all groups by the end of week 12 of implantation. Tb.Sp of the BCPG3 group was significantly higher than other groups throughout the study. These results showed that the introduction of $\mathrm{Sr}$ seemed not provide additional benefit to the osteogenesis of BCPG3 material in osteoporotic conditions.

\subsection{Histological analysis of osteoporotic bone regeneration}

Immunohistological staining of $\mathrm{Cxc19}$, Runx2 and RANKL proteins were conducted in the current study (Fig. 7). The positive expression of Cxc19 seemed to be less in BCPG3 and SrBCPG3 groups. Runx2 and RANKL positive expressions were more in BCPG3 group than others. The positive staining signals was further quantified in Fig. 7B. The result was in accordance with in vitro gene and protein expression and in vivo serum biomarker analysis. Furthermore, we observed from H\&E staining that more lacunae were presented in the BCPG3 and SrBCPG3 groups. The observation was further verified by counting lacuna number in different groups in a blind test. To be 
more specific, the lacunae in the BCP and SrBCP bone samples were presented preferentially on newly formed bone, not within the material. However, the lacunae in the BCPG3 and SrBCPG3 bone samples were more evenly distributed as they were found embedded in materials too. With SEM observation of the bone samples, it was possible to confirm that all bone paste materials were successfully integrated into the newly formed trabeculae without any gap observed at the boundary. Transition of the material to bone was demonstrated. A number of lacunae could be found within the BCPG3 material, which indicated that active bone remodeling had occurred inside the bone paste.

\section{DISCUSSION}

The development of organic/inorganic injectable materials for bone tissue repair has been gaining interest for their suitability to minimal invasive surgery. In addition to their suitable biocompatibility and bioactivity, these materials can be employed in bone injuries, even in those cases where there is limited access to the defect and where their filling may not result to be uniform. ${ }^{7,15}$ Different osteoconductive calcium phosphate or bioactive glass granules have been used with cohesive hydrogels to form injectable bone pastes. Several products existing on the market were developed according to this composition, i.e., CERASORB ${ }^{\circledR}$ Paste (Germany), n-IBS ${ }^{\circledR}$ (Portugal), ReproBone $^{\circledR}$ novo paste (UK) and Actifuse FLOW (USA). In this study, we successfully fabricated injectable bone pastes consisting of BCP nanoparticles and phosphoserine-tethered generation 3 poly(epsilon-lysine) dendron. 
The cohesive texture of bone pastes allows to improve the interactions between material and bone surfaces and the resistance to washout from bleeding of bone defects. Here, the doping of the pastes with $\mathrm{Sr}$ was investigated to ascertain whether it would significantly increase the amount of bone pastes being washed out in the PBS solution at $37^{\circ} \mathrm{C}$. The observed higher washout levels were compatible with a previous study which demonstrated that the degradation rate of the Sr-doped bioceramics was significantly faster than the pure bioceramics. ${ }^{29} \mathrm{Sr}$ replacement of $\mathrm{Ca}$ in the crystal lattice affected material stability. In an in vivo study, Cardemil et al. also reported that Sr-doped calcium phosphate granules had a faster degradation rate which failed to provide a stable surface for osteogenesis to occur. ${ }^{42}$ In the present work, the preservation of pastes from being washed out was investigated showing that biomaterials including G3-K PS reduced the leaking of material likely due to the ability of the phosphoserine moieties, presented at higher density through the dendrimer branching, to interact with the calcium phosphate crystal of the BCP nanoparticles thus acting as an electrostatic crosslinker. ${ }^{17}$ Bosko et al. studied the flow properties of dendrimers as compared to their linear counterparts. ${ }^{43}$ Spherical symmetry and highly constrained nature of the molecular architecture of dendrimers would result in viscoelastic properties that differ markedly from those of linear chain molecules. The symmetric monodisperse dendritic skeleton, like the G3-K PS used here, is much more in favor of constructing highly efficient gel systems compared with the linear analogue. ${ }^{44}$ And the viscoelastic response of dendrimers is also attributed to the enhanced correlations with increasing generation. ${ }^{45}$ Moreover, particle size and shape 
played an important role in the cohesion of the paste. Sohrabi et al. investigated the effect of bioactive glass particle size on rheological properties of the hyaluronic acid biocomposite paste. They discovered that at the same weight, the paste that comprised of smaller particles exhibits higher surfaces and hence the particles exhibit more friction together with polymer molecules that results in higher viscosity. ${ }^{46}$ This finding was also supported by a study conducted by Garcia et al. who characterized the rheological behavior of dense suspensions of calcium carbonate ground under various operating conditions. ${ }^{47}$ Their result indicated that the viscosity increases when the particle size decreases due to attractive interparticle forces. Ryabenkova et al. further investigated the effect of nanoscale HA particle morphology on rheological properties of the paste. ${ }^{48}$ Two paste-types were compared, with the HA particles of both types being rod shaped with a range of sizes between $20-80 \mathrm{~nm}$ while differing in the particle aspect ratio. The tests at a constant shear showed that the particles with a smaller aspect ratio was more viscous.

Cell viability and osteogenesis of the osteoporotic animal-derived MSCs grown on G3-K PS integrated pastes were elevated. This result was coherent with a study conducted by Galli et al. who generated a layer of G3-K PS film on grade 4 titanium surfaces. ${ }^{26}$ It was then used as a culture substrate for murine calvaria-derived osteoblastic cells and primary progenitor cells from bone marrow. Their results verified that the dendron-coated substrate increased gene expression of ALP and osteocalcin, in both osteoblastic cells and progenitor cells at days 3 and 6 of culture. Moreover, the mRNA levels of Wnt signaling related genes were also markedly increased. Similarly, 
Meikle et al., deposited solid-phase synthesized G3-K PS dendrons onto etched titanium oxide surfaces as a near-to-monolayer film. ${ }^{17}$ They demonstrated that the presence of G3-K PS monolayer was able to stimulate the precipitation of an uniform calcium phosphate phase after only 3 days of SBF treatment. Furthermore, this monolayer was able to stimulate osteoblast-like cells proliferation (i.e. MG63 and SAOS-2) at a level higher than control (etched titanium) and an improvement of differentiation degree for SAOS-2 cells at day 14 . These previous reports as well as the present study prove that the cell adhesion promoting poly(epsilon-lysine) dendron with functionalized phosphoserine group is able to support osteoprogenitor cell proliferation and differentiation. The integration of Sr presented a positive effect on cell viability of MSCs until day 3 and promoted the early Runx 2 osteogenic marker expression. This result was in line with a previous study which showed that porous Sr-loaded $\beta$-TCP based scaffold could significantly increase the gene expression of Runx 2 in the rat bone marrow derived MSCs at day 4, as compared to the non-loaded scaffold. ${ }^{49}$ Panzavolta et al. also found that Sr containing HA based scaffold enhanced osteoblast activity and reduced both number and differentiation of osteoclast from monocytes precursors. ${ }^{50}$ It was demonstrated by several preclinical studies that $\mathrm{Sr}$ element could improve osteoporotic bone strength, bone microarchitecture and fracture healing by enhancing pre-osteoblastic proliferation and inhibiting osteoclastic differentiation via extracellular regulated protein kinases (ERK) related pathways. ${ }^{51-53}$ However, no additional benefit was shown by Sr incorporation on cell viability at day 7 or ALP gene expression. 
In this study, it is for the first time reported that the G3-K PS carrier could downregulate $\mathrm{Cxc19}$ expression, as characterized by $\mathrm{PCR}$, in vivo serum ELISA and immunohistological staining of implanted sample seem to consistently demonstrate. Recently, Cxc19 was discovered as an angiostatic factor released by osteoblasts in the bone marrow microenvironment. Huang et al. as well as other investigators have shown that Cxc19 released by osteoblasts interacts with vascular endothelial growth factor (VEGF) and avoids its binding to endothelial cells and osteoblasts, thus inhibiting angiogenesis and osteogenesis both in mouse bone and in vitro model. ${ }^{41,54}$ The motivation why BCPG3 and SrBCPG3 groups demonstrated a suppressive effect on Cxc19 is currently unknown. It can be hypothesized that the phosphoserinefunctionalized branches of the dendrons might protect the VEGF from its undesired blocking thus enabling bone angiogenesis. Lode et al. investigated that the in vitro release of VEGF after physical adsorption to the unmodified bone cement as well as to cements modified with mineralized collagen I, O-phospho-L-serine and sodium citrate, respectively. ${ }^{55}$ Their results demonstrated that in the phosphoserine-modified cement groups, the bioactivity of released VEGF was much higher than other groups. They concluded that the phosphoserine modification of bone cement might support activation of angiogenesis by delivery of VEGF in a local and sustained manner. As demonstrated by H\&E staining, BCPG3 and SrBCPG3 groups presented more newly formed blood vessels embedded in the trabecular bone matrix. Vogt et al. demonstrated that the people affected by osteoporosis or osteopenia show a blood supply lower than people with normal bone mass, indicating that bone blood supply and bone mineral density are 
highly correlated. ${ }^{56}$ This result confirmed that stimulating angiogenesis in the bone microenvironment might be an important measure to prevent the osteoporosis disease. ${ }^{57}$ Here, western blotting results confirmed that $\mathrm{Cxc19}$ protein played an important role in osteogenesis induced by G3-K PS incorporated bone pastes. The supplementary of Cxc19 protein in cell culture medium would abolish the influence of BCPG3 and SrBCPG3 on osteogenic protein expressions.

In this study, the loading of $\mathrm{Sr}$ in bone pastes caused a decrease of RANKL expression, as determined by PCR, Western blotting analysis and immunohistological staining of implanted sample. RANKL signaling is important for terminal differentiation of monocytes/macrophages into osteoclasts to initiate bone resorption. Here, it was observed that serum bone resorption marker, CTX-I, was downregulated in Sr containing bone paste groups. Bone homeostasis is preserved by coordinated cycles of bone resorption and formation. The coupling signals, transmitted by osteoclasts to osteoblasts, drive the transition from bone resorption to formation. ${ }^{58}$ When bone resorption was inhibited, bone formation would be impaired too. Consistently, serum bone formation marker P1NP and osteogenic Runx2 protein expression was decreased in $\mathrm{Sr}$ containing bone paste groups. A suppressed bone formation by $\mathrm{Sr}$ may explain why BCPG3 group bone showed a higher bone regeneration effect than SrBCPG3, in terms of a higher BMD, BV/TV and a lower Tb.Sp. The present study suggests that, when compared to Sr, the integration of G3-K PS carrier might yield a more important effect in enhancing osteogenesis of the BCP under osteoporotic condition. 


\section{CONCLUSIONS}

In this study, an injectable bone paste material integrating BCP nanoparticles with G3-K PS carrier was successfully synthesized with or without the doping of Sr element into the BCP nanocrystals. Both in vitro and in vivo findings showed that the integration of G3-K PS would downregulate $\mathrm{Cxc19}$ gene and protein expressions to achieve an enhanced bone regeneration effect, with respect to a higher BMD, BV/TV and a lower Tb.Sp. While bone resorption related RANKL and CTX-I levels was decreased by the introduction of Sr to bone pastes, bone formation was also hindered to a certain extent, in terms of decreased Runx2 and P1NP expressions. No additional benefit to osteoporotic bone regenerating ability of BCPG3 material was found with $\mathrm{Sr}$ incorporation. Our results indicated that the BCPG3 bone paste can become a highperformance bone filler in the treatment of osteoporotic bone defects.

\section{AUTHOR INFORMATION}

\section{Corresponding Author}

*E-mail: xiaoyang114@scu.edu.cn (Xiao Yang).

*E-mail: mariagrazia.raucci@cnr.it

\section{Notes}

There are no conflicts to declare. 


\section{ACKNOWLEDGEMENTS}

This work was supported by National Natural Science Foundation of China (grant no. 81971755), PNR Aging Program (2012-2018), Sichuan Science and Technology Program (2020YFS0038), Sichuan Science and Technology Innovation Team of China (2019JDTD0008), Guike AA17204085-2, Young Elite Scientist Sponsorship Program by CAST (2019QNRC001), Fundamental Research Funds for the Central Universities. We thank Dr. Li Chen from the Analytical \& Testing Center of Sichuan University for assistance with micro-CT images and Xiaoli Zhu from the National Engineering Research Center for Biomaterials of Sichuan University for help of animal administration.

\section{SUPPORTING INFORMATION}

Effect of different bone pastes on hematology parameters of the implanted Sprague

Dawley rats.

New bone volume fraction (nBV/TV, \%) within the defected area at week 8 and week 12. 


\section{REFERENCES}

(1) Manchinasetty, N. V. L.; Sato, T.; Aizawa, M.; Madanagurusamy, S.; Kikuchi, M. Influences of Combined Supplementation of Calcium Citrate and Calcium Carbonate on Injectable and Anti-Washout Hydroxyapatite/Collagen Bone Paste Utilizing Sodium Alginate. Journal of the Ceramic Society of Japan 2017, 125, 579-583.

( 2 ) Kretlow, J. D.; Young, S.; Klouda, L.; Wong, M.; Mikos, A. G. Injectable Biomaterials for Regenerating Complex Craniofacial Tissues. Adv Mater 2009, 21, 3368-3393.

( 3 ) Larsson, S.; Hannink, G. Injectable Bone-Graft Substitutes: Current Products, Their Characteristics and Indications, and New Developments. Injury 2011, 42 Suppl 2, S30-S34.

(4) Tamimi, F.; Torres, J.; Lopez-Cabarcos, E.; Bassett, D. C.; Habibovic, P.; Luceron, E.; Barralet, J. E. Minimally Invasive Maxillofacial Vertical Bone Augmentation Using Brushite Based Cements. Biomaterials 2009, 30, 208-216.

( 5 ) Nur Maulida, H.; Hikmawati, D.; Budiatin, A. S. Injectable Bone Substitute Paste Based on Hydroxyapatite, Gelatin and Streptomycin for Spinal Tuberculosis. Journal of Spine 2015, 04.

(6) Panchbhavi.; K., V. Augmentation of Internal Fixation of Osteoporotic Ankle Fracture Using Injectable Bone Substitute. Techniques in Foot \& Ankle Surgery 2007, 6, 264-269.

(7) Borhan, S.; Hesaraki, S.; Behnamghader, A. A.; Ghasemi, E. Rheological Evaluations and In Vitro Studies of Injectable Bioactive Glass-Polycaprolactone-Sodium Alginate Composites. J Mater Sci Mater Med 2016, 27, 137.

( 8 ) Fu, S.; Ni, P.; Wang, B.; Chu, B.; Zheng, L.; Luo, F.; Luo, J.; Qian, Z. Injectable and ThermoSensitive PEG-PCL-PEG Copolymer/Collagen/n-HA Hydrogel Composite for Guided Bone Regeneration. Biomaterials 2012, 33, 4801-4809.

(9) Gutowska, A.; Jeong, B.; Jasionowski, M. Injectable Gels for Tissue Engineering. Anat Rec 2001, $263,342-349$.

(10) Giannoni, P.; Villa, F.; Cordazzo, C.; Zardi, L.; Fattori, P.; Quarto, R.; Fiorini, M. Rheological Properties,Bbiocompatibility and In Vivo Performance of New Hydrogel-Based Bone Fillers. Biomater Sci 2016, 4, 1691-1703.

(11) D'Este, M.; Eglin, D. Hydrogels in Calcium Phosphate Moldable and Injectable Bone Substitutes: Sticky Excipients or Advanced 3-D Carriers? Acta Biomater 2013, 9, 5421-5430.

( 12 ) Laschke, M. W.; Witt, K.; Pohlemann, T.; Menger, M. D. Injectable Nanocrystalline Hydroxyapatite Paste for Bone Substitution: In Vivo Analysis of Biocompatibility and Vascularization. J Biomed Mater Res B Appl Biomater 2007, 82, 494-505.

(13) Raucci, M. G.; Fasolino, I.; Pastore, S. G.; Soriente, A.; Capeletti, L. B.; Dessuy, M. B.; Giannini, C.; Schrekker, H. S.; Ambrosio, L. Antimicrobial Imidazolium Ionic Liquids for the Development of Minimal Invasive Calcium Phosphate-Based Bionanocomposites. ACS Appl Mater Interfaces 2018, 10, 42766-42776.

(14) Cui, X.; Zhang, Y.; Wang, H.; Gu, Y.; Li, L.; Zhou, J.; Zhao, S.; Huang, W.; Zhou, N.; Wang, D.; Pan, H.; Rahaman, M. N. An Injectable Borate Bioactive Glass Cement for Bone Repair: Preparation, Bioactivity and Setting Mechanism. Journal of Non-Crystalline Solids 2016, 432, 150-157.

(15) Salehi, G.; Behnamghader, A.; Hesaraki, S.; Mozafari, M. Synergistic Effects of Carbohydrate Polymers on the Performance of Hybrid Injectable Bone Pastes. European Polymer Journal 2019, 119, 523-530. 
(16) Wei, X.; Egawa, S.; Matsumoto, R.; Yasuda, H.; Hirai, K.; Yoshii, T.; Okawa, A.; Nakajima, T.; Sotome, S. Augmentation of Fracture Healing by Hydroxyapatite/Collagen Paste and Bone Morphogenetic Protein-2 Evaluated Using a Rat Femur Osteotomy Model. J Orthop Res 2018, 36, 129137.

( 17 ) Meikle, S. T.; Bianchi, G.; Olivier, G.; Santin, M. Osteoconductive Phosphoserine-modified Poly( $\varepsilon$-Lysine) Dendrons: Synthesis, Titanium Oxide Surface Functionalization and Response of Osteoblast-like Cell Lines. J R Soc Interface 2013, 10, 20120765.

( 18 ) Wang, J.; Cooper, R. C.; He, H.; Li, B.; Yang, H. Polyamidoamine Dendrimer Microgels: Hierarchical Arrangement of Dendrimers into Micrometer Domains with Expanded Structural Features for Programmable Drug Delivery and Release. Macromolecules 2018, 51, 6111-6118.

( 19 ) Yellepeddi, V. K.; Ghandehari, H. Poly(amidoamine) Dendrimers in Oral Delivery. Tissue Barriers 2016, 4, e1173773.

(20) Hegde, N.; Velingkar, V.; Prabhakar, B. An Update on Design and Pharmacology of Dendritic Poly(L-Lysine). International Journal of Peptide Research and Therapeutics 2018, 25, 1539-1562.

(21) Raucci, M. G.; Alvarez-Perez, M. A.; Meikle, S.; Ambrosio, L.; Santin, M. Poly(Epsilon-Lysine) Dendrons Tethered with Phosphoserine Increase Mesenchymal Stem Cell Differentiation Potential of Calcium Phosphate Gels. Tissue Eng Part A 2014, 20, 474-485.

(22) Shcharbin, D.; Janaszewska, A.; Klajnert-Maculewicz, B.; Ziemba, B.; Dzmitruk, V.; Halets, I.; Loznikova, S.; Shcharbina, N.; Milowska, K.; Ionov, M.; Shakhbazau, A.; Bryszewska, M. How to Study Dendrimers and Dendriplexes III. Biodistribution, Pharmacokinetics and Toxicity In Vivo. J Control Release 2014, 181, 40-52.

(23) Zhang, H.; Zhou, Y.; Yu, N.; Ma, H.; Wang, K.; Liu, J.; Zhang, W.; Cai, Z.; He, Y. Construction of Vascularized Tissue-Engineered Bone with Polylysine-Modified Coral Hydroxyapatite and a Double Cell-Sheet Complex to Repair a Large Radius Bone Defect in Rabbits. Acta Biomater 2019, 91, 82-98.

(24) Reinstorf, A.; Ruhnow, M.; Gelinsky, M.; Pompe, W.; Hempel, U.; Wenzel, K.-W.; Simon, P. Phosphoserine-a Convenient Compound for Modification of Calcium Phosphate Bone Cement Collagen Composites. Journal of Materials Science: Materials in Medicine 2005, 15, 451-455.

( 25 ) Salgado, C. L.; Teixeira, B. I. B.; Monteiro, F. J. M. Biomimetic Composite Scaffold With Phosphoserine Signaling for Bone Tissue Engineering Application. Front Bioeng Biotechnol 2019, 7 , 206.

(26) Galli, C.; Piemontese, M.; Meikle, S. T.; Santin, M.; Macaluso, G. M.; Passeri, G. Biomimetic Coating with Phosphoserine-Tethered Poly(Epsilon-Lysine) Dendrons on Titanium Surfaces Enhances Wnt and Osteoblastic Differentiation. Clin Oral Implants Res 2014, 25, e133-e139.

(27) Zhao, R.; Chen, S.; Yuan, B.; Chen, X.; Yang, X.; Song, Y.; Tang, H.; Yang, X.; Zhu, X.; Zhang, $\mathrm{X}$. Healing of Osteoporotic Bone Defects by Micro-/Nano-Structured Calcium Phosphate Bioceramics. Nanoscale 2019, 11, 2721-2732.

(28) Zhu, Y.; Zhang, K.; Zhao, R.; Ye, X.; Chen, X.; Xiao, Z.; Yang, X.; Zhu, X.; Zhang, K.; Fan, Y.; Zhang, X. Bone Regeneration with Micro/Nano Hybrid-Structured Biphasic Calcium Phosphate Bioceramics at Segmental Bone Defect and the Induced Immunoregulation of MSCs. Biomaterials 2017, 147, 133-144.

(29) Zhao, R.; Chen, S.; Zhao, W.; Yang, L.; Yuan, B.; Ioan, V. S.; Iulian, A. V.; Yang, X.; Zhu, X.; Zhang, X. A Bioceramic Scaffold Composed of Strontium-Doped Three-Dimensional Hydroxyapatite Whiskers for Enhanced Bone Regeneration in Osteoporotic Defects. Theranostics 2020, 10, 1572-1589. 
( 30 ) Yuan, B.; Raucci, M. G.; Fan, Y.; Zhu, X.; Yang, X.; Zhang, X.; Santin, M.; Ambrosio, L. Injectable Strontium-doped Hydroxyapatite Integrated with Phosphoserine-Tethered Poly(EpsilonLysine) Dendrons for Osteoporotic Bone Defect Repair. Journal of Materials Chemistry B 2018, 6, 7974 7984.

(31) Lourenco, A. H.; Torres, A. L.; Vasconcelos, D. P.; Ribeiro-Machado, C.; Barbosa, J. N.; Barbosa, M. A.; Barrias, C. C.; Ribeiro, C. C. Osteogenic, Anti-Osteoclastogenic and Immunomodulatory Properties of a Strontium-Releasing Hybrid Scaffold for Bone Repair. Materials Science Engineering C 2019, 99, 1289-1303.

( 32 ) Meininger, S.; Moseke, C.; Spatz, K.; März, E.; Blum, C.; Ewald, A.; Vorndran, E. Effect of Strontium Substitution on the Material Properties and Osteogenic Potential of 3D Powder Printed Magnesium Phosphate Scaffolds. Materials Science Engineering C 2019, 98, 1145-1158.

(33) Zeng., J.; Guo., J.; Sun., Z.; Deng., F.; Xie., Y. Osteoblastic and Anti-Osteoclastic Activities of Strontium-Substituted Silicocarnotite Ceramics: In Vitro and In Vivo Studies. Bioactive Materials 2020, $5,435-446$.

( 34 ) Raucci, M. G.; Giugliano, D.; Alvarez-Perez, M. A.; Ambrosio, L. Effects on Growth and Osteogenic Differentiation of Mesenchymal Stem Cells by the Strontium-Added Sol-Gel Hydroxyapatite Gel Materials. J Mater Sci Mater Med 2015, 26, 90.

( 35 ) Xu, C.; Wang, X.; Zhou, J.; Huan, Z.; Chang, J. Bioactive Tricalcium Silicate/Alginate Composite Bone Cements with Enhanced Physicochemical Properties. J Biomed Mater Res B Appl Biomater 2018, 106, 237-244.

( 36 ) Zhang, Y.; Wei, L.; Chang, J.; Miron, R. J.; Shi, B.; Yi, S.; Wu, C. Strontium-Incorporated Mesoporous Bioactive Glass Scaffolds Stimulating In Vitro Proliferation and Differentiation of Bone Marrow Stromal Cells and In Vivo Regeneration of Osteoporotic Bone Defects. Journal of Materials Chemistry B 2013, 1, 5711-5722.

(37) Chen, X.; Wang, J.; Chen, Y.; Cai, H.; Yang, X.; Zhu, X.; Fan, Y.; Zhang, X. Roles of Calcium Phosphate-Mediated Integrin Expression and MAPK Signaling Pathways in the Osteoblastic Differentiation of Mesenchymal Stem Cells. Journal of Materials Chemistry B 2016, 4, 2280-2289.

( 38 ) Yang, L.; Kong, J.; Qiu, Z.; Shang, T.; Chen, S.; Zhao, R.; Raucci, M. G.; Yang, X.; Wu, Z. Mineralized Collagen-Modified PMMA Cement Enhances Bone Integration and Reduces Fibrous Encapsulation in the Treatment of Lumbar Degenerative Disc Disease. Regenerative Biomaterials 2019, $7,1-13$.

(39) Zhao, R.; Xie, P.; Zhang, K.; Tang, Z.; Chen, X.; Zhu, X.; Fan, Y.; Yang, X.; Zhang, X. Selective Effect of Hydroxyapatite Nanoparticles on Osteoporotic and Healthy Bone Formation Correlates with Intracellular Calcium Homeostasis Regulation. Acta Biomater 2017, 59, 338-350.

( 40 ) Basu, S.; Ghosh, A.; Barui, A.; Basu, B. (Fe/Sr) Codoped Biphasic Calcium Phosphate with Tailored Osteoblast Cell Functionality. ACS Biomaterials Science \& Engineering 2018, 4, 857-871.

(41) Huang, B.; Wang, W.; Li, Q.; Wang, Z.; Yan, B.; Zhang, Z.; Wang, L.; Huang, M.; Jia, C.; Lu, J.; Liu, S.; Chen, H.; Li, M.; Cai, D.; Jiang, Y.; Jin, D.; Bai, X. Osteoblasts Secrete Cxc19 to Regulate Angiogenesis in Bone. Nat. Commun. 2016, 7, 13885.

( 42 ) Cardemil, C.; Elgali, I.; Xia, W.; Emanuelsson, L.; Norlindh, B.; Omar, O.; Thomsen, P. Strontium-Doped Calcium Phosphate and Hydroxyapatite Granules Promote Different Inflammatory and Bone Remodelling Responses in Normal and Ovariectomised Rats. PLoS One 2013, 8, e84932.

(43) Bosko, J. T.; Todd, B. D.; Sadus, R. J. Viscoelastic Properties of Dendrimers in the Melt from Nonequlibrium Molecular Dynamics. Journal of Chemical Physics 2005, 121, 12050-12059. 
(44) Feng., Y.; Liu., Z. X.; Chen., H.; Yan., Z. C.; He., Y. M.; Liu., C. Y.; Fan., Q. H. A Systematic Study of Peripherally Multiple Aromatic Ester-Functionalized Poly(benzyl ether) Dendrons for the Fabrication of Organogels: Structure - Property Relationships and Thixotropic Property. Chemistry 2014, 20, 7069-7082.

(45) Costanzo, S.; Scherz, L. F.; Schweizer, T.; Krger, M.; Floudas, G.; Schlüter, A. D.; Vlassopoulos, D. Rheology and Packing of Dendronized Polymers. Macromolecules 2016, 49, 7054-7068.

( 46 ) Sohrabi, M.; Hesaraki, S.; Kazemzadeh, A.; Alizadeh, M. Development of Injectable Biocomposites from Hyaluronic Acid and Bioactive Glass Nano-Particles Obtained from Different SolGel Routes. Materials Science Engineering C 2013, 33, 3730-3744.

( 47 ) Garcia., F.; Bolay., N. L.; Frances., C. Rheological Behaviour and Related Granulometric Properties of Dense Aggregated Suspensions During an Ultrafine Comminution Process. Powder Technology 2003, 130, 407-414.

( 48 ) Y.Ryabenkova.; A.Pinnock.; P.A.Quadros.; R.L.Goodchild.; G.Möbus.; A.Crawford.; P.V.Hatton.; C.A.Miller. The Relationship Between Particle Morphology and Rheological Properties in Injectable Nano-Hydroxyapatite Bone Graft Substitutes. Materials Science and Engineering: C 2017, 75, 1083-1090.

(49) Ge., C.; Chen., F.; Mao., L.; Liang., Q.; Liu., C. Strontium Ranelate-Loaded POFC/ $\beta$-TCP Porous Scaffolds for Osteoporotic Bone Repair. RSC Advances 2020, 10, 9016-9025.

( 50 ) Silvia, P.; Paola, T.; Sonia, C.; Annapaola, P.; Milena, F.; Adriana, B. Strontium-Substituted Hydroxyapatite-Gelatin Biomimetic Scaffolds Modulate Bone Cell Response. Macromolecular Bioscience 2018, 18, 1800096.

(51) Schumacher, M.; Wagner, A. S.; Kokesch-Himmelreich, J.; Bernhardt, A.; Rohnke, M.; Wenisch, S.; Gelinsky, M. Strontium Substitution in Apatitic CaP Cements Effectively Attenuates Osteoclastic Resorption but Does not Inhibit Osteoclastogenesis. Acta Biomaterialia 2016, 184-194.

( 52 ) Mi, B.; Xiong, W.; Xu, N.; Guan, H.; Fang, Z.; Liao, H.; Zhang, Y.; Gao, B.; Xiao, X.; Fu, J. Strontium-Loaded Titania Nanotube Arrays Repress Osteoclast Differentiation through Multiple Signalling Pathways: In Vitro and In Vivo Studies. Scientific Reports 2017, 7, 2328.

( 53 ) Zhou, Y.; Guan, X.; Liu, T.; Wang, X.; Yu, M.; Yang, G.; Wang, H. Whole Body Vibration Improves Osseointegration by Up-Regulating Osteoblastic Activity but Down-Regulating OsteoblastMediated Osteoclastogenesis via ERK1/2 Pathway. Bone 2015, 71, 17-24.

(54) Wu, J.; Wang, A.; Wang, X.; Li, G.; Jia, P.; Shen, G.; Chen, B.; Yuan, Y.; Zhang, H.; Yang, F.; $\mathrm{Xu}, \mathrm{Y}$. Rapamycin Improves Bone Mass in High-Turnover Osteoporosis with Iron Accumulation through Positive Effects on Osteogenesis and Angiogenesis. Bone 2019, 121, 16-28.

( 55 ) Lode, A.; Wolf-Brandstetter, C.; Reinstorf, A.; Bernhardt, A.; Konig, U.; Pompe, W.; Gelinsky, M. Calcium Phosphate Bone Cements, Functionalized with VEGF: Release Kinetics and Biological Activity. J Biomed Mater Res A 2007, 81, 474-483.

(56) Vogt, M. T.; Cauley, J. A.; Kuller, L. H.; Nevitt, M. C. Bone Mineral Density and Blood Flow to the Lower Extremities: the Study of Osteoporotic Fractures. Journal of Bone and Mineral Research 1997, 12, 283-289.

(57) Tong, X.; Chen, X.; Zhang, S.; Huang, M.; Shen, X.; Xu, J.; Zou, J. The Effect of Exercise on the Prevention of Osteoporosis and Bone Angiogenesis. Biomed Res Int 2019, 2019, 8171897.

(58) Ikebuchi, Y.; Aoki, S.; Honma, M.; Hayashi, M.; Sugamori, Y.; Khan, M.; Kariya, Y.; Kato, G.; Tabata, Y.; Penninger, J. M.; Udagawa, N.; Aoki, K.; Suzuki, H. Coupling of Bone Resorption and Formation by RANKL Reverse Signalling. Nature 2018, 561, 195-200. 
Table 1 Commercialized injectable bone materials: classification, properties and indications.

\begin{tabular}{|c|c|c|c|c|}
\hline Classification & Product brand & Solidification time & Mechanical strength & Indications and use \\
\hline \multirow{8}{*}{$\begin{array}{c}\text { PMMA } \\
\text { Based } \\
\text {-Bioinert, load-bearing, non- } \\
\text { degradable, bone regenerates } \\
\text { around the implant }\end{array}$} & Osteopal V & $17-18 \min$ & $85-95 \mathrm{MPa}$ & $\begin{array}{l}\text { compression fractures of the vertebral body, vertebral body tumors, } \\
\text { symptomatic vertebral haemangiomas, etc. }\end{array}$ \\
\hline & SpinePlex & $9-10 \mathrm{~min}$ & $100-106 \mathrm{MPa}$ & vertebral compression fractures, etc. \\
\hline & Confidence & $>10 \mathrm{~min}$ & NR & vertebroplasty and kyphoplasty, etc. \\
\hline & Osteobond & NR & NR & internal fixation and artificial joint replacement, etc. \\
\hline & DePuy CMW1 & NR & $107 \mathrm{MPa}$ & hip resurfacing arthroplasty, etc. \\
\hline & Simplex P & $>9 \mathrm{~min}$ & $100 \mathrm{MPa}$ & hip, knee, and shoulder implant fixation, etc. \\
\hline & $\begin{array}{l}\text { VersaBond Bone } \\
\text { Cement }\end{array}$ & NR & NR & knee and hip replacement surgery, etc. \\
\hline & Mendec Cranio & NR & NR & cranial gap repair, etc. \\
\hline \multirow{3}{*}{$\begin{array}{l}\text { Calcium sulphate based } \\
\text {-Bioactive, Non-load- } \\
\text { bearing, material degradation } \\
\text { accompanied with new bone } \\
\text { formation }\end{array}$} & geneX & $15 \mathrm{~min}$ & 9-12 MPa & tibial plateau fractures, long-bone non-unions, etc. \\
\hline & MIIGTMX3 & NR & NR & internal fixation of pedicle screw system, etc. \\
\hline & STIMULAN $®$ & $4 \mathrm{~min}$ & 9-12 MPa & infected long bone nonunions, etc. \\
\hline \multirow{5}{*}{$\begin{array}{l}\text { Calcium phosphate based } \\
\text {-Bioactive, Non-load- } \\
\text { bearing, material degradation } \\
\text { accompanied with new bone } \\
\text { formation }\end{array}$} & In'Oss ${ }^{\text {TM }}$ Putty & NA & new bone to support & $\begin{array}{l}\text { filling or reconstruction of osseous bone defects or gaps of the } \\
\text { skeletal system, etc. }\end{array}$ \\
\hline & $\begin{array}{l}\text { CERAMENT }^{\mathrm{TM}} \\
\text { BONE VOID } \\
\text { FILLER }\end{array}$ & NA & new bone to support & $\begin{array}{l}\text { filling or reconstruction of osseous bone defects or gaps of the } \\
\text { skeletal system, etc. }\end{array}$ \\
\hline & n-IBS $®$ & NA & new bone to support & $\begin{array}{l}\text { Reconstruction of post traumatic bone defects, periodontal defects } \\
\text { filling, alveolar bone filling, filling cages in spinal surgery, } \\
\text { acetabulum reconstruction, etc. }\end{array}$ \\
\hline & rebone & $15-18 \min$ & $\begin{array}{l}\text { compressive } \\
\text { strength }>35 \mathrm{MPa}\end{array}$ & compression fractures of the vertebral body, vertebral tumors, etc. \\
\hline & ReproBone ${ }^{\circledR}$ novo & NA & new bone to support & $\begin{array}{l}\text { filling or reconstruction of osseous bone defects, filling cages in } \\
\text { spinal surgery, etc. }\end{array}$ \\
\hline
\end{tabular}




\begin{tabular}{|c|c|c|c|c|}
\hline & $\begin{array}{l}\text { NANO HA } \\
\text { PASTE }\end{array}$ & NA & new bone to support & $\begin{array}{l}\text { filling or reconstruction of osseous bone defects, filling cages in } \\
\text { spinal surgery, acetabulum reconstruction, etc. }\end{array}$ \\
\hline & $\begin{array}{l}\text { EXABONE® } \\
\text { Paste }\end{array}$ & NA & new bone to support & $\begin{array}{c}\text { filling or reconstruction of osseous bone defects, filling cages in } \\
\text { spinal surgery, acetabulum reconstruction, etc. }\end{array}$ \\
\hline & Actifuse Flow & NA & new bone to support & spine and limbs, etc. \\
\hline \multirow{3}{*}{$\begin{array}{c}\text { Bioglass based } \\
\text { - Bioactive, Non-load- } \\
\text { bearing, material degradation } \\
\text { accompanied with new bone } \\
\text { formation }\end{array}$} & BonAlive $\AA$ putty & NA & new bone to support & bony voids and gaps, etc. \\
\hline & BioSphere Putty & NA & new bone to support & anterior cervical decompression and fusion, etc. \\
\hline & NovaBone putty & NA & new bone to support & alveolar bone defects, etc. \\
\hline
\end{tabular}

NR: not reported in the product pamphlet or website;

NA: not applicable -- no solidification. 


\section{Figure captions}
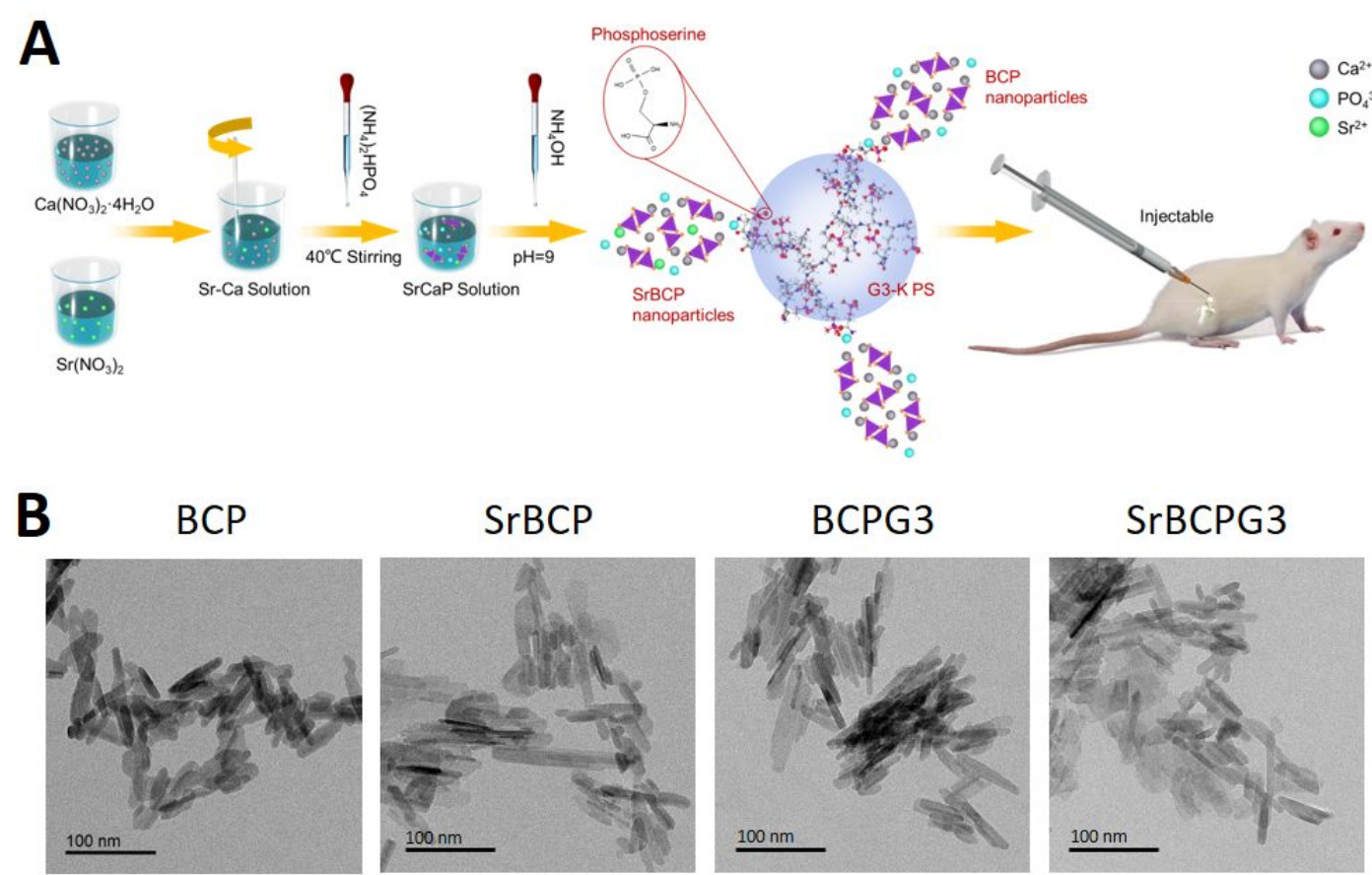

C
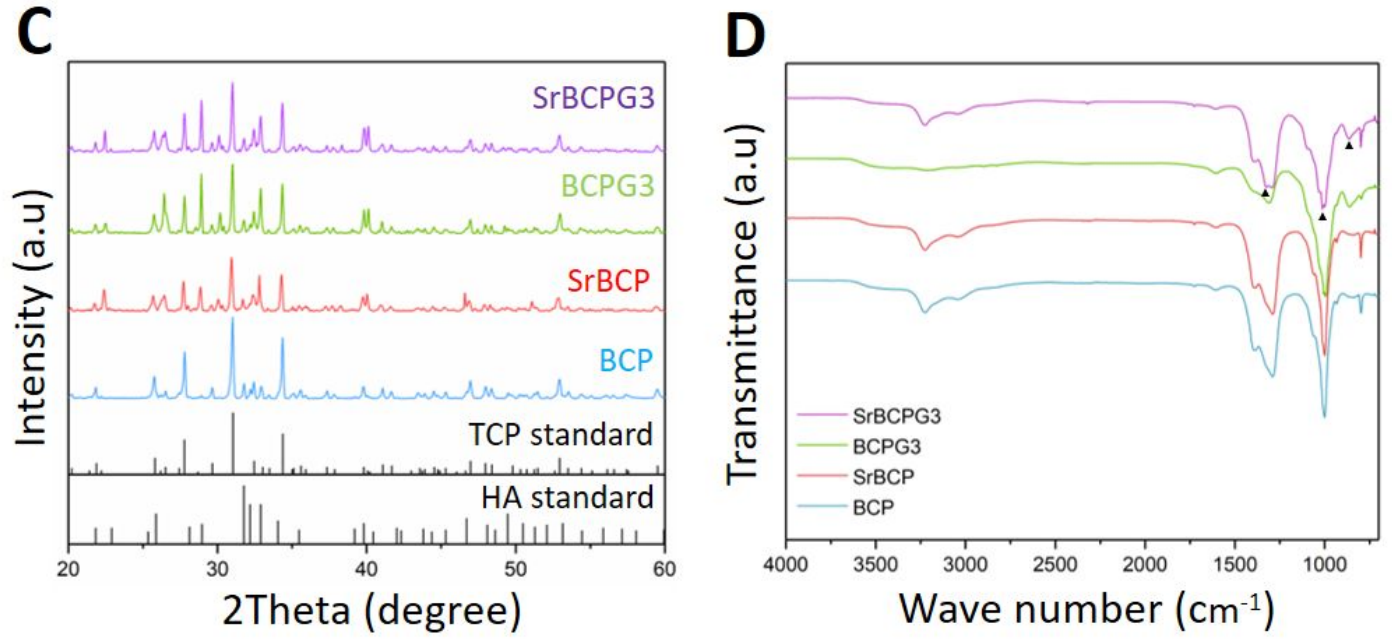

Fig. 1 Fabrication and physiochemical properties of the injectable bone pastes. (A) The schematic diagram of the main steps of preparing G3-K PS integrated BCP containing bone pastes for treating osteoporotic bone defect. (B) TEM images, (C) XRD patterns and (D) FTIR spectra of the BCP, SrBCP, BCPG3 and SrBCPG3 bone pastes; arrowheads indicate amide band. 
A

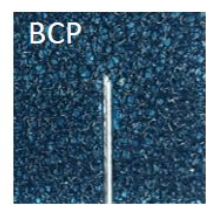

SrBCP
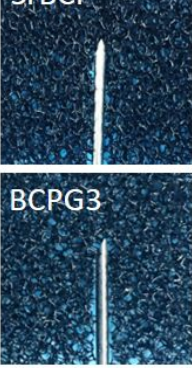

$S r B C P$ G3

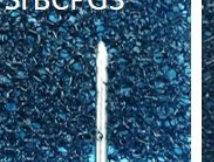

$\mathbf{C}_{250}$
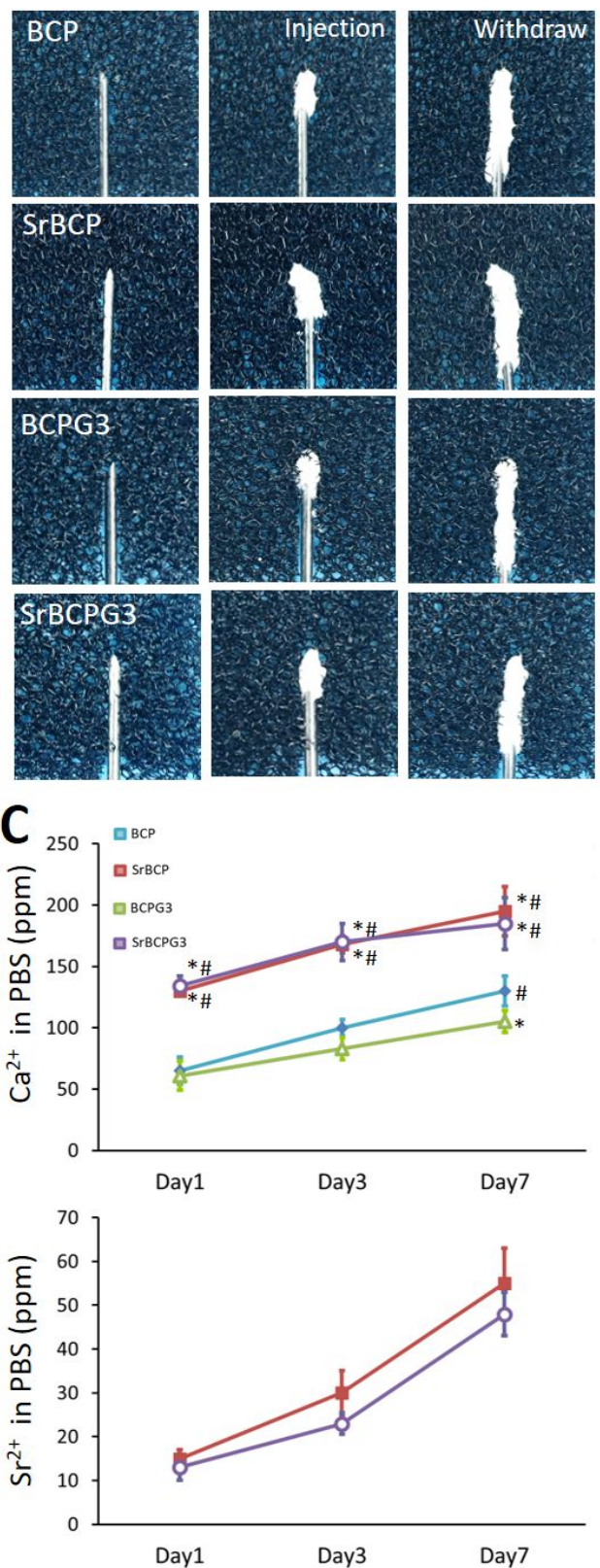

B
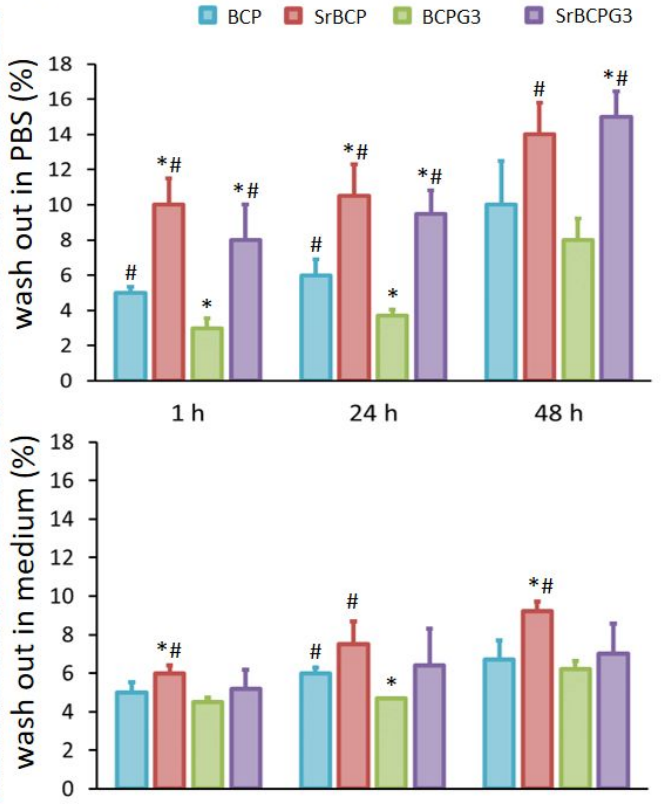

$1 \mathrm{~h}$
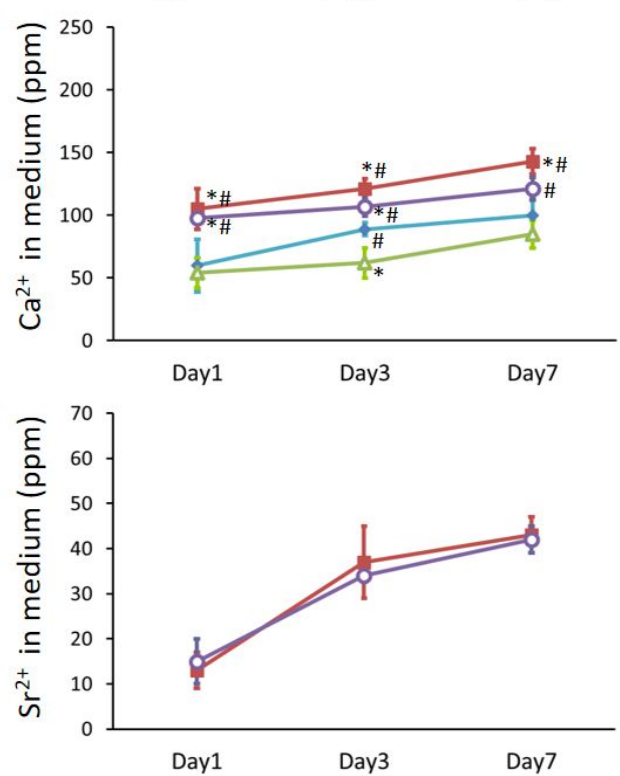

Fig. 2 (A) Photographic illustration and characterization of the retention of BCP, SrBCP, BCPG3 and SrBCPG3 bone pastes in polystyrene sponge at $37{ }^{\circ} \mathrm{C}$. (B) The amount of different bone pastes being washed out (\%) after immersing into PBS or cell culture medium at $37^{\circ} \mathrm{C}$ for $1 \mathrm{~h}, 24 \mathrm{~h}$ and $48 \mathrm{~h}$. (C) Ion release profile of different bone pastes immersed in PBS or culture medium. *Significant difference from BCP group with $\mathrm{p}<$ 0.05; \#Significant difference from BCPG3 group with $\mathrm{p}<0.05$. 
A

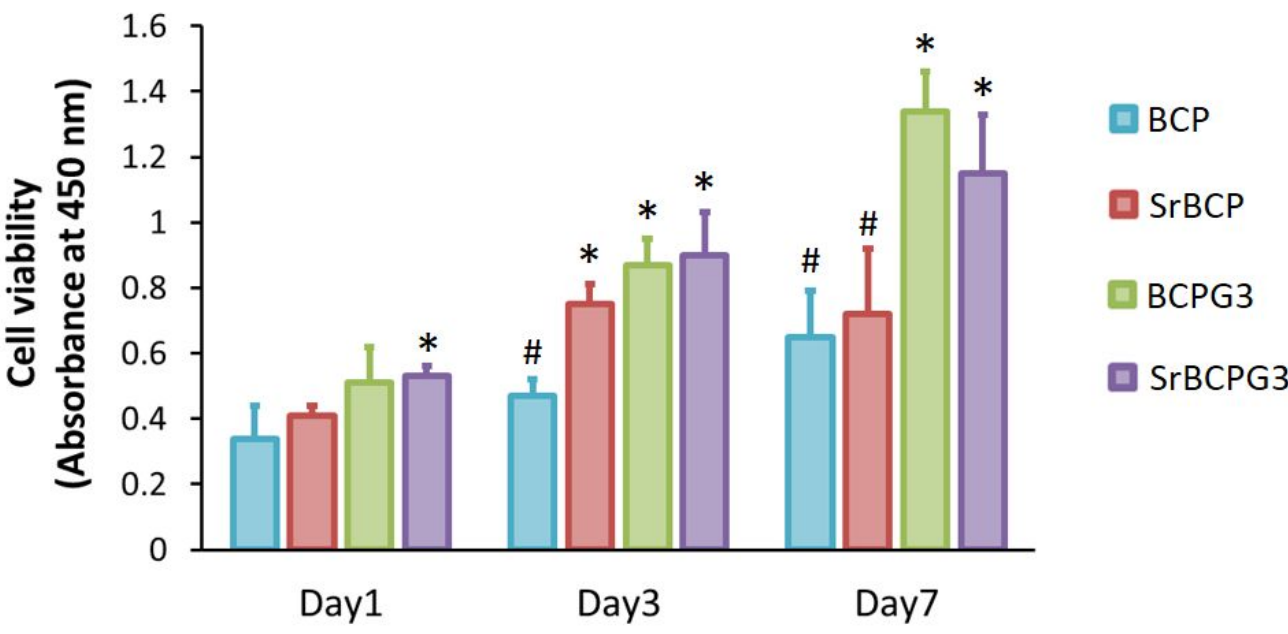

B
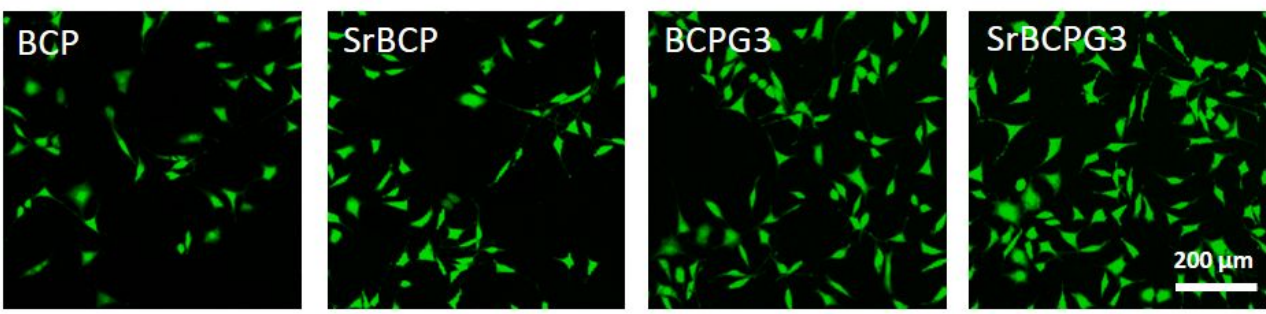

C
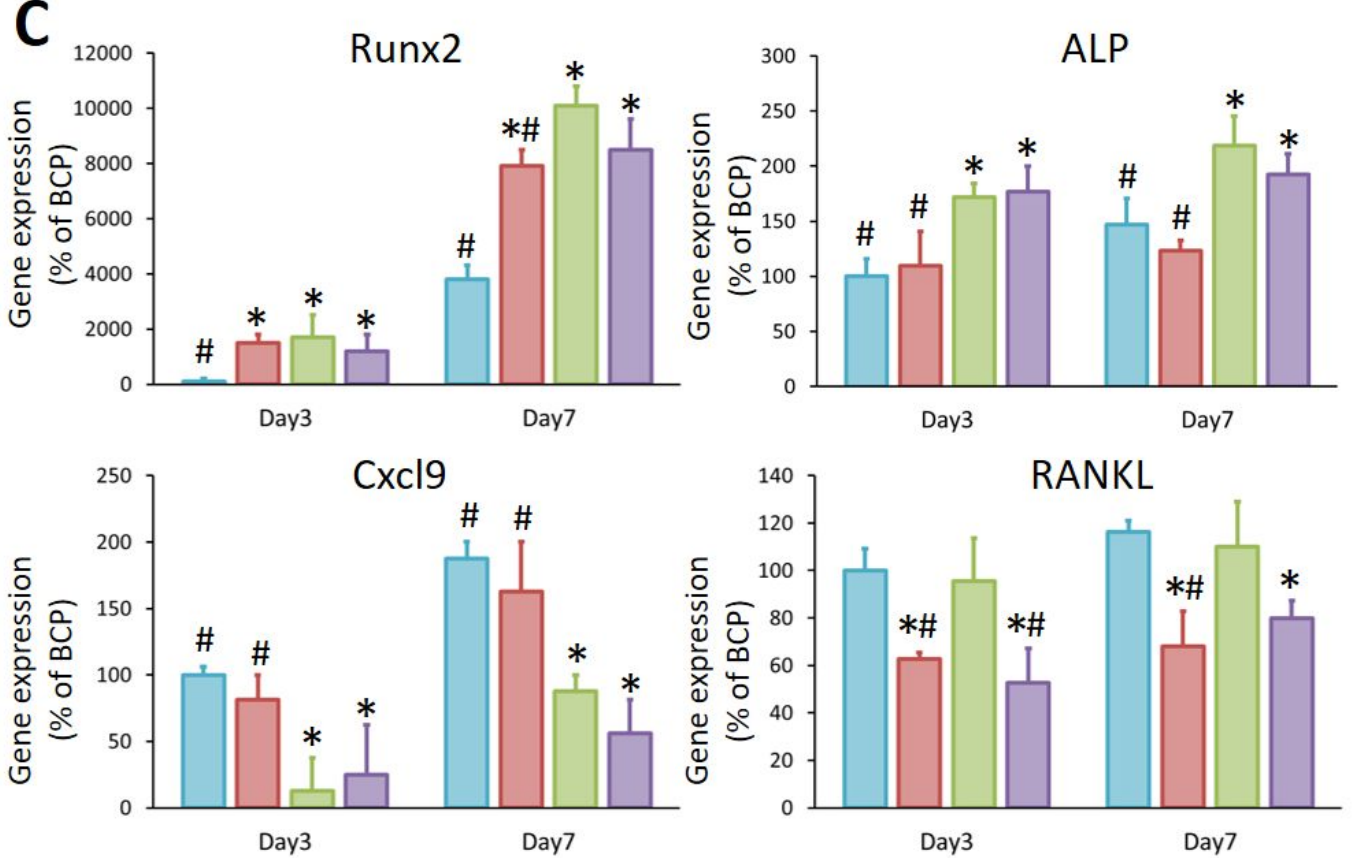

Fig. 3 (A) Cell counting kit-8 assay for MSCs cell viability cocultured with BCP, SrBCP, BCPG3 and SrBCPG3 bone pastes for 1, 3 and 7 days; MSCs used were primarily isolated from osteoporotic rat bone. (B) Confocal laser scanning microscopy observations of MSCs grown on different bone pastes. (C) qRT-PCR analysis of the gene expression levels of Runx2, ALP, Cxc19 and RANKL in MSCs cocultured with 
different bone pastes at days 3 and 7. *Significant difference from BCP group with $\mathrm{p}<$ 0.05; \#Significant difference from BCPG3 group with $\mathrm{p}<0.05$.

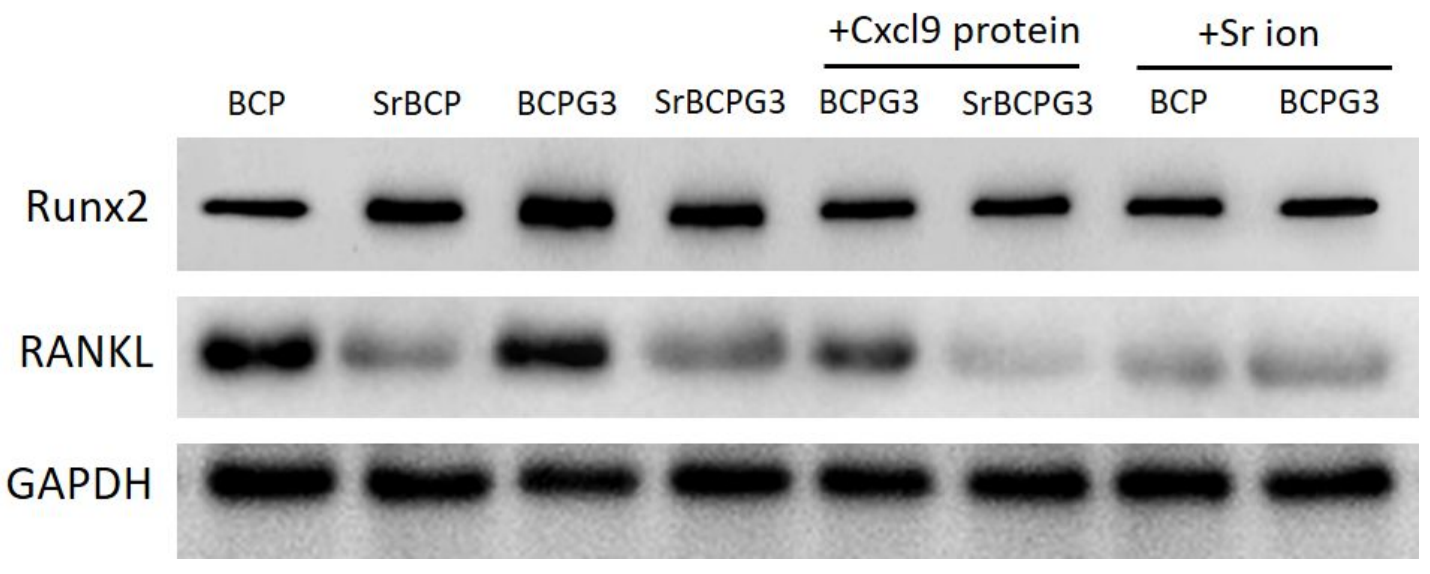

Fig. 4 Representative Western blotting analysis for Runx2, RANKL and GAPDH protein expressions in MSCs cocultured with BCP, SrBCP, BCPG3 and SrBCPG3 bone pastes. $+\mathrm{Cxc19}$ : additional $\mathrm{Cxc19}$ protein $\left(250 \mathrm{ng} \mathrm{mL}^{-1}\right)$ was added into the cell culture medium of BCPG3 and SrBCP groups; + Sr ion: additional $\mathrm{Sr}$ ion $(3 \mathrm{mM})$ added into the cell culture medium of BCP and BCPG3 groups. 

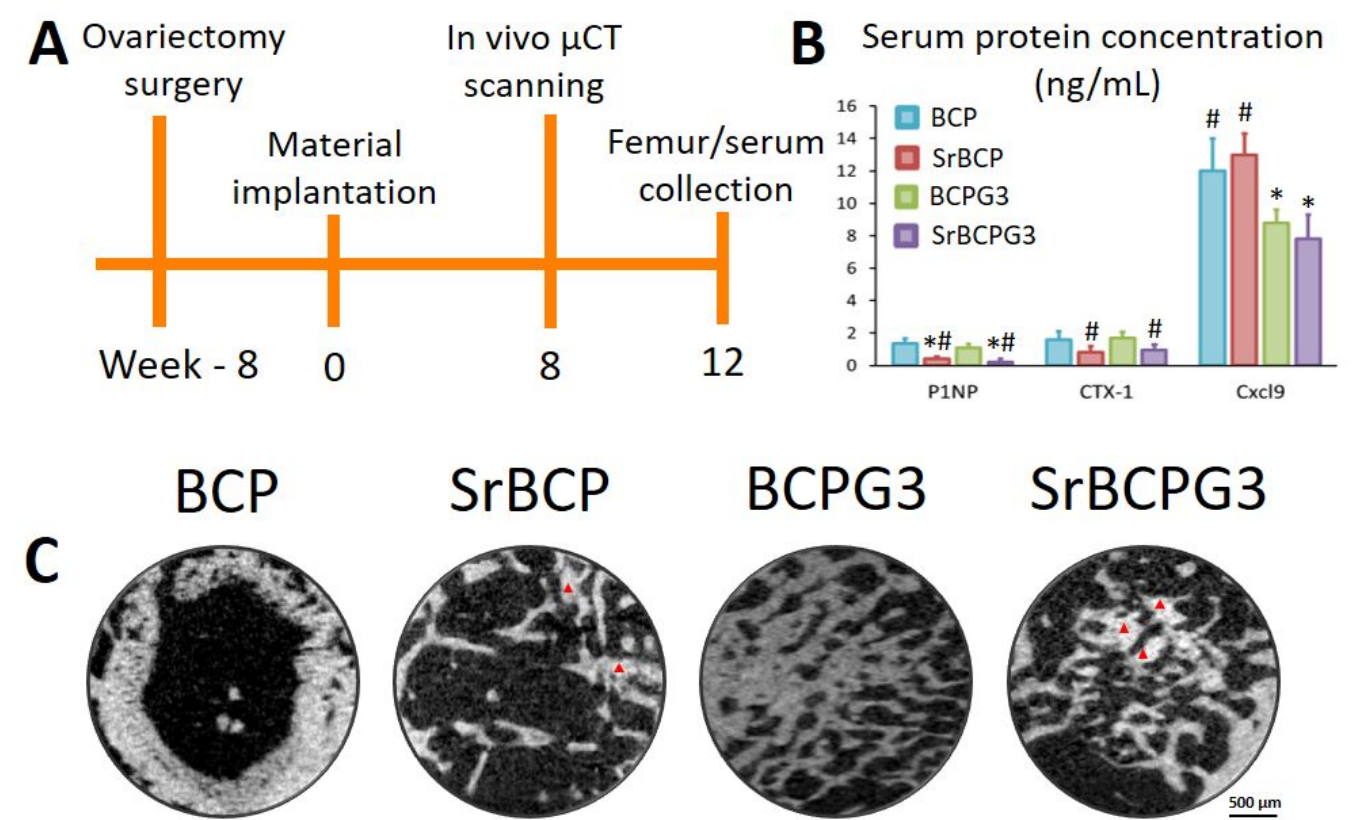

BCPG3

SrBCPG3
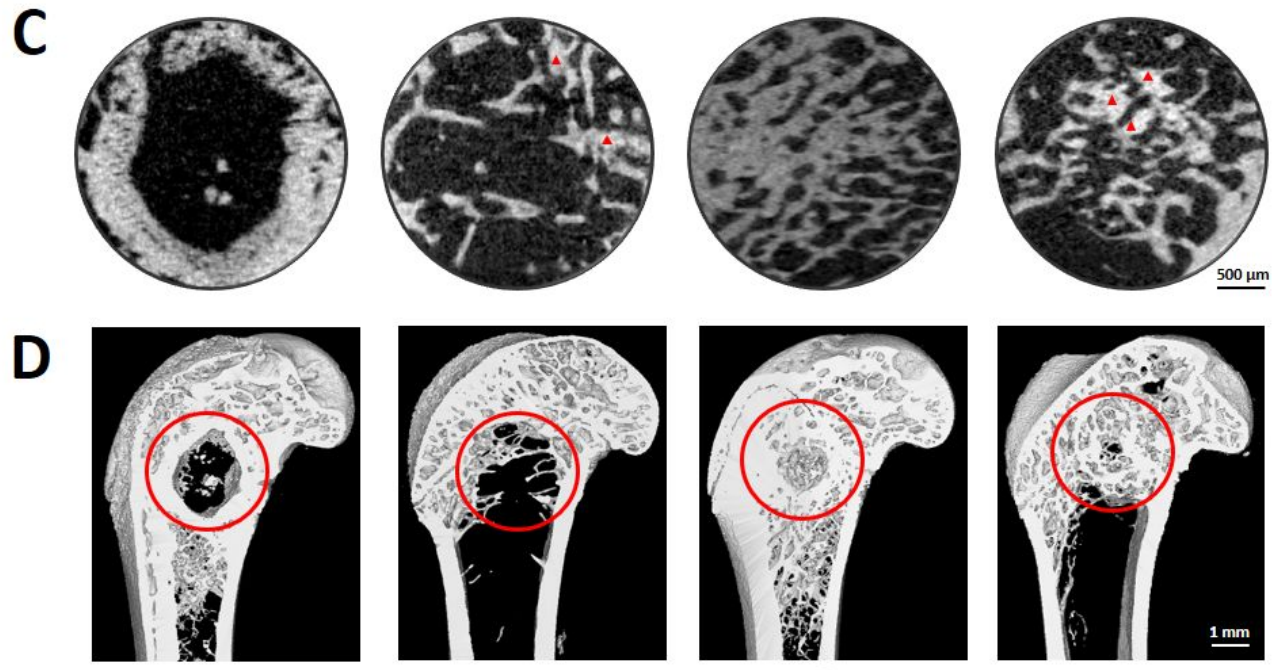

E
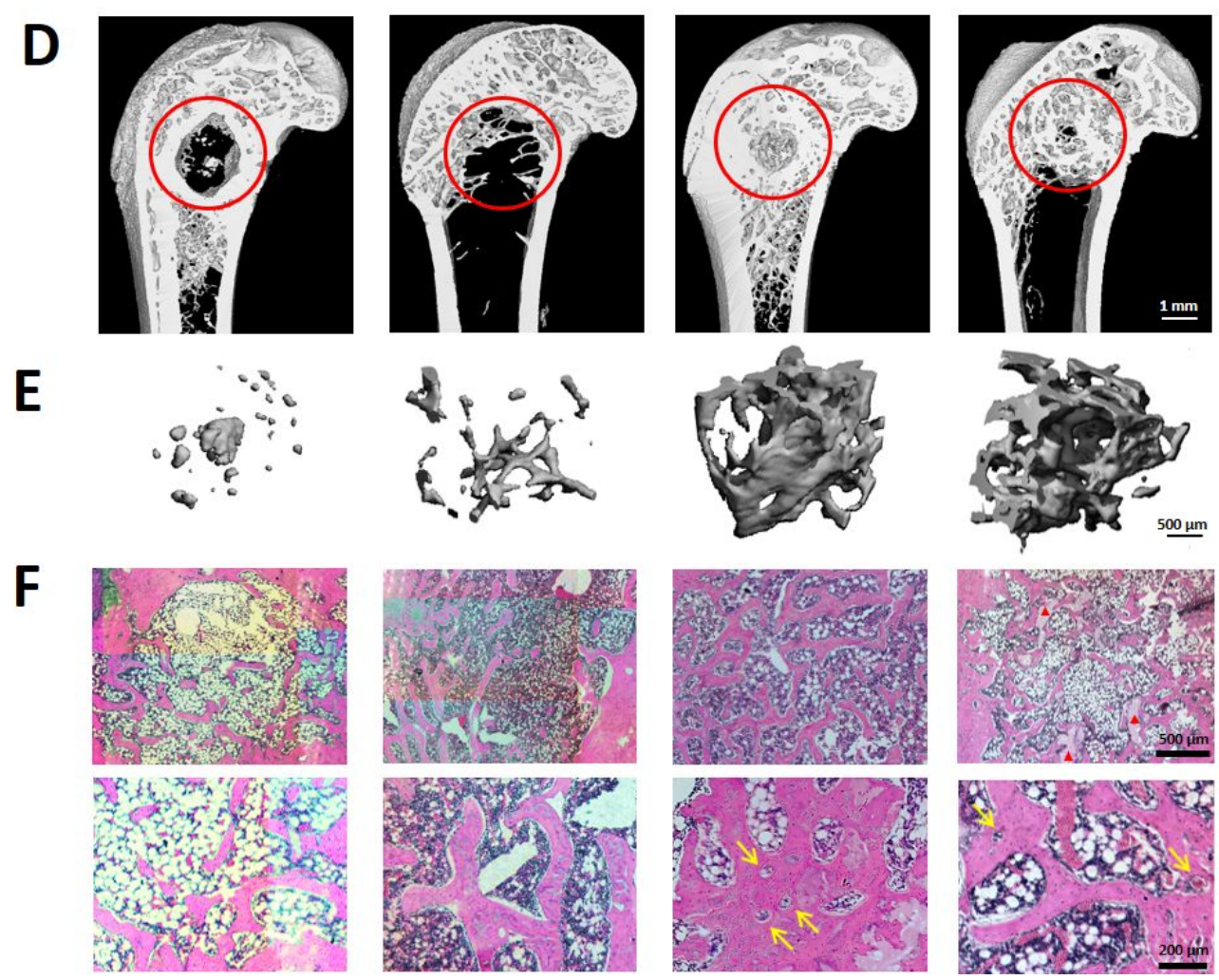

Fig. 5 Serum analysis and visualization of osteoporotic bone repair by different bone pastes. (A) The Design and timeline of the in vivo study to evaluate bone paste materials implanted in ovariectomized rat bone defect. (B) Serum levels of bone formation marker P1NP, resorption marker CTX-I and chemokine Cxc19 of respective groups, * Significant difference from BCP group with $\mathrm{p}<0.05$; \#Significant difference from 
BCPG3 group with $\mathrm{p}<0.05$. (C) Representative tomographic images demonstrating the current working threshold to segment the non-degraded material (red arrow head) embedded in newly formed bone after implantation. (D) Reconstructed micro-CT images of the coronal sections from the metaphyseal femur at week 12 (red circles indicate new bone formation within a $3 \mathrm{~mm}$ diameter defect). (E) 3D reconstruction of newly formed bone inside the defected area. (F) Hematoxylin and eosin (H\&E) stained histological sections of different groups (red arrowheads indicate non-degraded material; yellow arrow: newly formed blood vessels in bone).

A

Week 8

Week 12

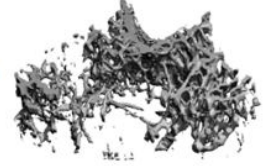

BCP

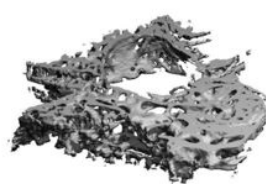

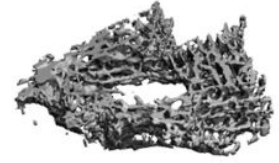
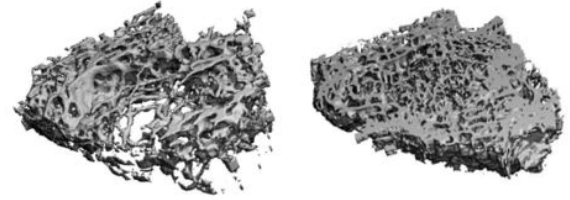

SrBCPG3
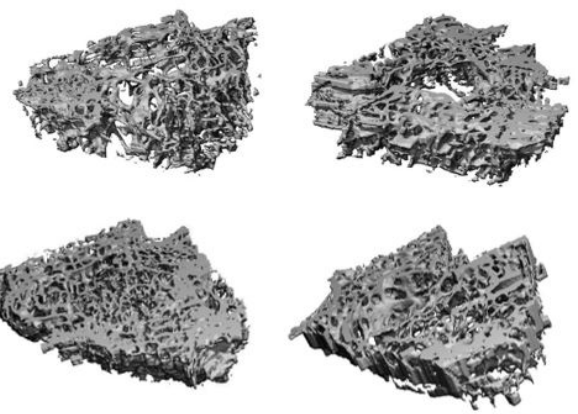

$1 \mathrm{~mm}$
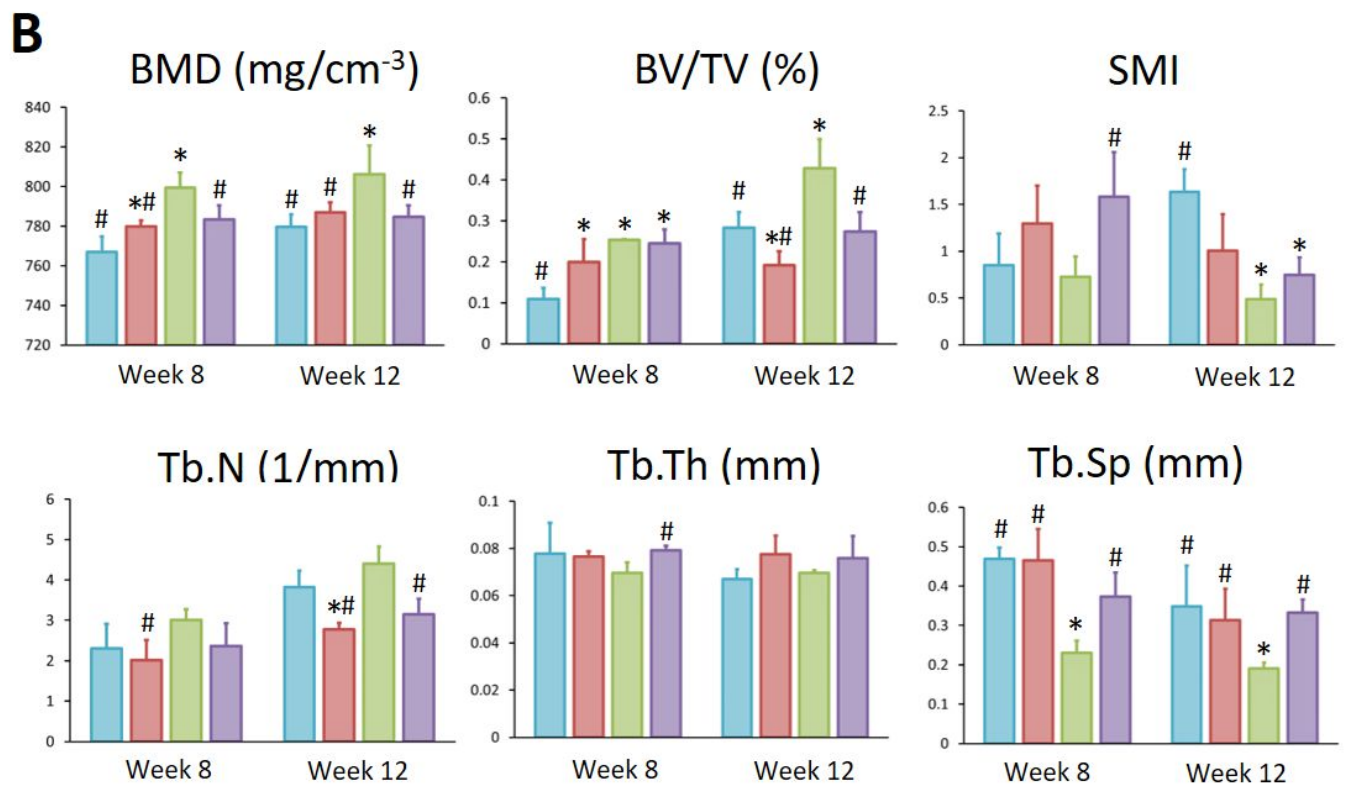
Fig. 6 (A) The three-dimensionally reconstruction of the trabecular network at week 8 and week 12 of different groups. (B) Trabecular microarchitecture parameters within the region of interest, including bone mineral density (BMD, $\mathrm{mg} / \mathrm{cm}^{-3}$ ), bone volume fraction (BV/TV, \%), structural model index (SMI), trabecular number (Tb.N, 1/mm), trabecular thickness (Tb.Th, mm) and trabecular separation (Tb.Sp, mm). *Significant difference from BCP group with $\mathrm{p}<0.05$; \#Significant difference from BCPG3 group with $\mathrm{p}<0.05$.
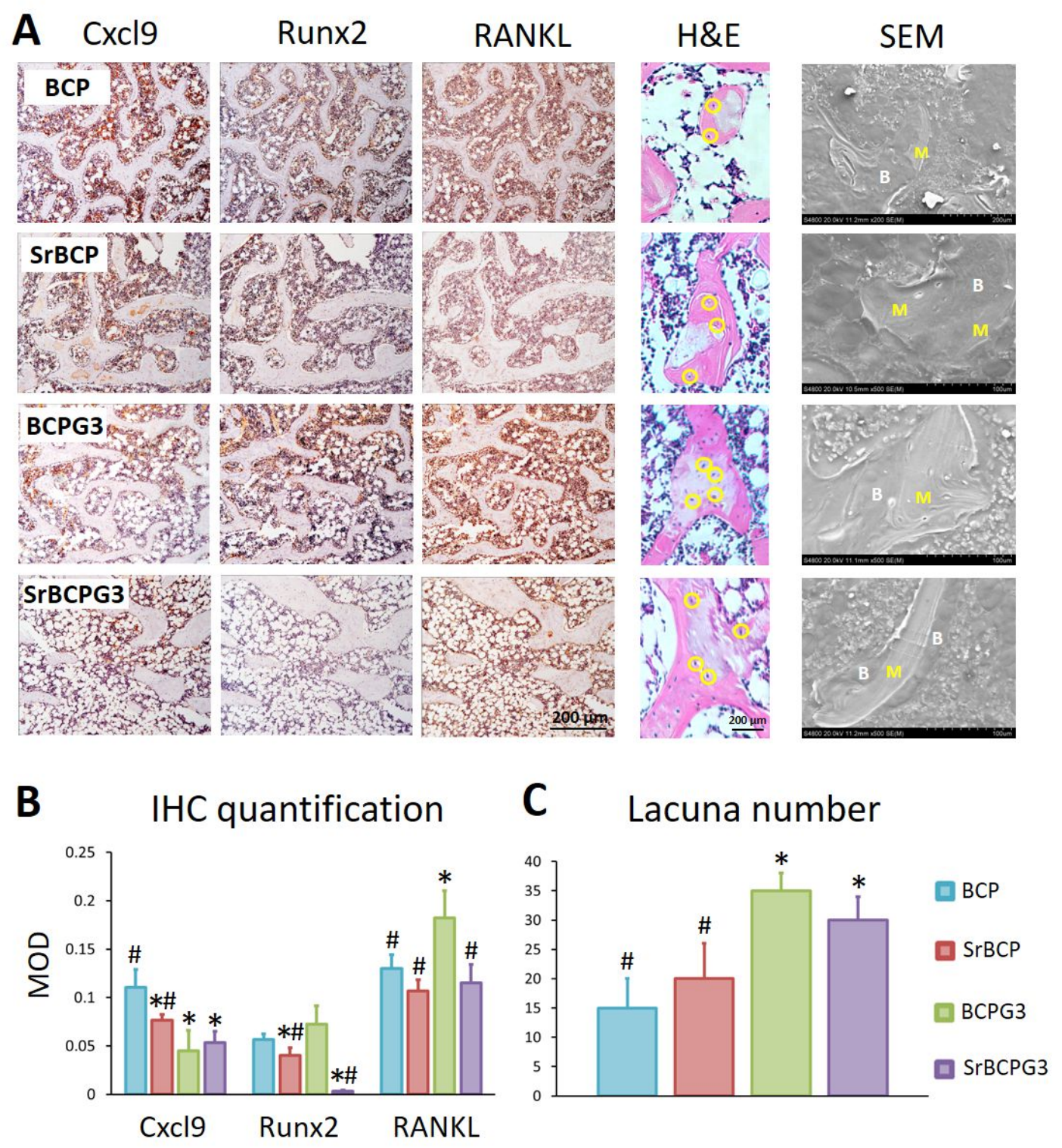
Fig. 7 Histological analysis for osteoporotic bone defect regeneration with different bone pastes. (A) Left: Consecutive slices of the 12-week implanted samples from different groups showing Cxc19, Runx2 and RANKL protein expressions (brown); Middle: H\&E staining of representative single trabeculae from different groups (yellow circle indicates lacuna); Right: SEM observation of the single trabeculae (M: nondegraded material; B: new bone). (B) Mean optical density (MOD) of Cxc19, Runx2 and RANKL positive staining of different groups. (C) Quantified lacuna number in each group. *Significant difference from BCP group with $\mathrm{p}<0.05$; \#Significant difference from BCPG3 group with $\mathrm{p}<0.05$. 